\title{
Decoherence and oscillations of supernova neutrinos
}

\author{
Jörn Kersten ${ }^{1, a} \mathbb{D}^{\mathbb{D}}$, Alexei Yu. Smirnov ${ }^{2,3, b}$ \\ ${ }^{1}$ Institute for Physics and Technology, University of Bergen, Postboks 7803, 5020 Bergen, Norway \\ ${ }^{2}$ Max-Planck-Institut für Kernphysik, Saupfercheckweg 1, 69117 Heidelberg, Germany \\ ${ }^{3}$ The Abdus Salam ICTP, Strada Costiera 11, 34014 Trieste, Italy
}

Received: 9 February 2016 / Accepted: 9 June 2016 / Published online: 20 June 2016

(C) The Author(s) 2016. This article is published with open access at Springerlink.com

\begin{abstract}
Supernova neutrinos have several exceptional features which can lead to interesting physical consequences. At the production point their wave packets have an extremely small size $\sigma_{x} \sim 10^{-11} \mathrm{~cm}$; hence the energy uncertainty can be as large as the energy itself, $\sigma_{E} \sim E$, and the coherence length is short. On the way to the Earth the wave packets of mass eigenstates spread to macroscopic sizes and separate. Inside the Earth the mass eigenstates split into eigenstates in matter and oscillate again. The coherence length in the Earth is comparable with the radius of the Earth. We explore these features and their consequences. (1) We present new estimates of the wave packet size. (2) We consider the decoherence condition for the case of wave packets with spatial spread and show that it is not modified by the spread. (3) We study the coherence of neutrinos propagating in a multilayer medium with density jumps at the borders of layers. In this case coherence can be partially restored due to a "catchup effect", increasing the coherence length beyond the usual estimate. This catch-up effect can occur for supernova neutrinos as they cross the shock wave fronts in the exploding star or the core of the Earth.
\end{abstract}

\section{Introduction}

Detecting the neutrino burst from a galactic supernova will be one of the major and outstanding scientific events of this century. It will bring about an enormous amount of new physics information both on the dynamics of the collapse as well as the explosion and on neutrinos themselves. Hence, a deep understanding of the underlying processes and effects is a must.

\footnotetext{
a e-mail: joern.kersten@uib.no

b e-mail: smirnov@ictp.it
}

Supernovae - the strongest known sources of neutrinos - provide a unique environment for the production and the flavor evolution of neutrino states. In addition to the standard resonant flavor conversion in matter [1], the huge neutrino density makes neutrino-neutrino interactions relevant [2-4] that can lead to various collective effects: synchronized oscillations [3,5], bipolar oscillations [6,7], spectral splits or swaps [8-11], self-induced parametric resonance [12], etc. (see, e.g., [13-15] for reviews). Stimulated flavor transitions can occur due to turbulence in a medium [16].

Given the special conditions with very high temperature and density in the neutrinosphere, where neutrinos are produced, a very short time scale for the microscopic production processes is realized. Consequently, the neutrino states are described by very short wave packets in configuration space. Indeed, previous estimates of the wave packet size were $\sigma_{x}=1.8 \cdot 10^{-14} \mathrm{~cm}$ for neutrinos produced in the core of the protoneutron star [17] and $\sigma_{x}=4.2 \cdot 10^{-9} \mathrm{~cm}$ for neutrinos emitted at a radius of $1000 \mathrm{~km} \mathrm{[18]} \mathrm{and} \mathrm{thus} \mathrm{in} \mathrm{a}$ region with much lower matter density. These calculations used Coulomb scattering to determine the mean free path of electrons inside the supernova.

Another important feature is the huge distance supernova neutrinos travel from a star to the Earth. Both these features, very short wave packets and very long baselines, affect the flavor evolution of the neutrino states and consequently observations. In fact, two effects happen:

- shift of the wave packets of the eigenstates by $\Delta x_{\text {shift }}$ due to the difference of group velocities and eventually their separation when $\Delta x_{\text {shift }}>\sigma_{x}$, implying a loss of coherence of the neutrino states, and

- spread of wave packets of individual eigenstates due to the presence of different energies in a wave packet.

Due to the very small $\sigma_{x}$, decoherence may occur at small distances inside the supernova, even before the region of collective effects. Due to the huge distance to the Earth, the sep- 
aration and spread of the packets become macroscopic. So, in principle, one can discuss the possibility of time tagging the wave packets.

Propagation decoherence has been studied mainly in vacuum [19-27], but also in matter [18,28-31] and in dense neutrino gases [32]. It is characterized by the coherence length $L_{\text {coh }}-$ the distance at which $\Delta x_{\text {shift }} \sim \sigma_{x}$. For distances larger than $L_{\mathrm{coh}}$, the eigenstates no longer interfere because their wave packets no longer overlap. Thus, the oscillatory pattern disappears and the oscillation probability becomes baseline-independent.

The coherence is also affected by the detection process. In particular, coherence can be restored by an accurate energy measurement [20], which was confirmed via a quantum field theory calculation [33]. In this case the detector must have a coherent observation time larger than the difference of the arrival times of two packets $\Delta t_{\text {det }}$ or (equivalently) a sufficiently good energy resolution, $\Delta E_{\text {res }}<1 / \Delta t_{\text {det }}$. Thus, observable effects depend on characteristics of the detector: coherent time of observation and energy resolution.

The wave packets give the complete picture of the evolution of neutrino states in configuration space. However, in order to determine observational results it is enough to treat the problem in the energy representation, which simplifies the considerations substantially. The results of the energymomentum and configuration-space treatments are equivalenct as far as observations are concerned, at least in the stationary source approximation $[20,34]$ and if no time tagging is performed.

The effects of source and detector are symmetric and can be described by an effective wave packet which includes characteristics of both source and detector. In fact, information about the wave packet of the source is included in the generated energy spectrum and information about the one of the detector in the detector's energy resolution.

It is believed that propagation decoherence does not affect the dynamics of the flavor evolution. Observable effects are then determined essentially by the energy spectrum at production, flavor evolution without decoherence, and the energy resolution of the detector. As mentioned above, however, the separation of supernova neutrinos is very fast due to their very short wave packet size. This could affect the dynamics of the flavor evolution in the region of collective effects [35], although a tentative answer was negative. In contrast, in [36] it was claimed that decoherence does influence collective oscillations (essentially due to the non-linear character of the problem) and lead to non-trivial flavor transformations.

Propagation decoherence is a reversible process; no information is lost in a system when wave packets separate. Hence, coherence can be restored by further propagation in matter if the difference of group velocities changes sign or (as mentioned above) in the detector.
The consideration in configuration space may have some advantage in the case of a complicated matter profile. It helps to obtain a clear physics interpretation of the results of integrating over energy. We will consider in detail the evolution of the wave packets all the way from the production point to a detector, focussing on the consequences of the small wave packet size of supernova neutrinos. We will study decoherence and partial restoration of coherence. New interesting effects are realized in oscillations in the matter of the Earth, which are related to an accidental coincidence of the coherence length and the size of the Earth. Some preliminary results have been published in [35].

Apart from separating, the wave packets also spread, since they comprise waves with different energies [37,38]. The increase of the size of a packet depends on the absolute values of neutrino masses. Effects of the spread on oscillations (which were not explored extensively before) are among the main objectives of this work.

The paper is organized as follows. In Sect. 2 we consider the production of neutrinos in a supernova and present an improved estimate of their wave packet size. We consider propagation decoherence, generalizing the vacuum results to propagation in matter. In Sect. 3 we consider decoherence in the energy-momentum representation. We show the equivalence of separation of the packets in configuration space and energy averaging. In Sect. 4 we study flavor evolution and coherence in a multi-layer medium where neutrino states split at each border between layers. We describe the "catchup effect" - partial restoration of the coherence between certain components of the split states. In Sect. 5 we discuss the spread of wave packets both in vacuum and in matter. We show that the coherence conditions for wave packets with and without spread coincide. In Sect. 6 we apply our results to supernova neutrino oscillations in the matter of the Earth.

\section{Supernova neutrino wave packets}

\subsection{Size of the wave packet}

The characteristics of the neutrino wave packets (WP) produced in a supernova depend on the phase of the explosion, since physical conditions and contributing processes change. We will consider different phases in order.

\subsubsection{Neutronization burst}

During the earliest stage of a supernova, mainly electron neutrinos are produced by electron capture, $p e^{-} \rightarrow n v_{e}$. We assume the nucleons to be localized well enough that the size of their WP is negligible (see below). Then the time scale for the electron capture process is given by the interval of time during which the electron WP crosses the proton 
[20]: $\tau \simeq \sigma_{x}^{e} / v^{e} \simeq \sigma_{x}^{e}$ for relativistic electrons. Here $\sigma_{x}^{e}$ is the size of the electron WP in configuration space and $v_{e}$ is the electron velocity. During this time a neutrino is emitted coherently. Consequently, its WP has a size $\sigma_{x} \simeq \tau \simeq \sigma_{x}^{e}$.

In turn, we estimate the electron WP size as $\sigma_{x}^{e} \simeq \lambda_{e}$, where $\lambda_{e}$ is the mean free path between two collisions of an electron that change its momentum by more than the momentum uncertainty $\sigma_{p}^{e} \simeq 1 / \sigma_{x}^{e}$. Collisions with a smaller momentum transfer correspond to forward scattering, which does not modify the WP and thus cannot localize a state. Introducing the electron fraction $Y_{e}$ we have $n_{e} \simeq Y_{e} n_{N}$, where $n_{N}$ is the nucleon number density, which is related to the mass density $\rho$ by $n_{N} \simeq N_{A} \rho /\left(1 \mathrm{~g} \cdot \mathrm{mol}^{-1}\right)$, where $N_{A}$ is the Avogadro constant. We neglect the Pauli blocking and consider only electron-electron as well as electronproton scattering. We expect electron-positron and Compton scattering to be subdominant due to the significantly smaller densities of the respective scattering partners. Approximating the cross section for ee scattering by the result in the center-of-mass frame and the one for ep scattering by the result for infinitely heavy protons we obtain

$\sigma\left(\Delta p>\sigma_{p}^{e}\right) \simeq \frac{4 \pi \alpha^{2}}{\sigma_{p}^{e 2}}$

for both processes. Then

$\frac{1}{\lambda_{e}} \simeq \frac{8 \pi \alpha^{2}}{\sigma_{p}^{e 2}} \cdot Y_{e} n_{N} \simeq 8 \pi \alpha^{2} \sigma_{x}^{e 2} Y_{e} n_{N}$

since the electron and proton number densities are roughly equal. Using $\sigma_{x}^{e} \simeq \lambda_{e}$ we obtain the total width of the neutrino $\mathrm{WP} \sigma_{x} \simeq \sigma_{x}^{e}$ :

$\sigma_{x} \simeq\left(8 \pi \alpha^{2} Y_{e} n_{N}\right)^{-1 / 3} \simeq 1.4 \cdot 10^{-11} \mathrm{~cm}\left(\frac{10^{12} \mathrm{~g} / \mathrm{cm}^{3}}{2 Y_{e} \rho}\right)^{1 / 3}$.

Note that the third root of the density and cross-section enters this expression, so the order of magnitude of the result should be correct despite our rather crude approximations. For a density $\rho=\left(10^{11}-10^{12}\right) \mathrm{g} / \mathrm{cm}^{3}$ and an electron fraction $Y_{e}=0.5$ in the production region (neutrinosphere) we obtain

$\sigma_{x} \simeq(1.4-3) \cdot 10^{-11} \mathrm{~cm}$,

or in energy-momentum space

$\sigma_{E} \simeq \frac{1}{\sigma_{x}} \simeq(0.7-1.5) \mathrm{MeV}$

\footnotetext{
${ }^{1}$ We define the WP width as the position or energy uncertainty of a particle. We employ the "intermediate wave packet" picture for the neutrinos. This approach produces the correct results for oscillation probabilities, as shown by the quantum field theory treatment of neutrino oscillations in vacuum $[33,37,39]$. We consider ultra-relativistic neutrinos throughout.
}

Notice that these values do not depend on the neutrino energy. Incidentally, the thermal wavelength, which has been suggested as an alternative measure of the neutrino position uncertainty [20], yields a similar value,

$\sigma_{x} \sim \lambda_{T}=\frac{2 \pi}{\langle p\rangle} \sim \frac{2 \pi}{3 T} \sim 8 \cdot 10^{-12} \mathrm{~cm}$

for $T \sim 5 \mathrm{MeV}$.

For nucleons, which are non-relativistic, we estimate the size of WP from the thermal wavelength

$\sigma_{x}^{N} \sim \lambda_{T}^{N} \sim \frac{2 \pi}{\sqrt{3 m_{N} T}} \sim 10^{-12} \mathrm{~cm}$,

which is of the same order of magnitude as the mean distance between nucleons $d \sim n_{N}^{-1 / 3} \sim(1-3) \cdot 10^{-12} \mathrm{~cm}$ and much smaller than the width of the electron WP. Hence, the nucleon WP size does not play a role in our discussion, justifying our initial assumption. Note that the magnetic fields inside a supernova are too weak to change the momentum of an electron between two collisions significantly, so they do not influence the WP size.

\subsubsection{Accretion and cooling phase}

For $v_{e}$, electron capture remains the main production process. Consequently, the WP size is still given by Eq. (3). Compared to the neutronization burst, the physical parameters in the neutrinosphere change in opposite directions: while the matter density $\rho$ increases, the electron fraction $Y_{e}$ decreases by about an order of magnitude [40,41]. As a result, we expect the $v_{e}$ WP size to increase moderately.

Electron antineutrinos $\bar{v}_{e}$ are mainly produced by positron capture on neutrons, $n e^{+} \rightarrow p \bar{v}_{e}$, so their $\sigma_{x}$ is equal to the WP size of the positron. The size of the positron WP can be estimated in the same way as that of the electron WP. The most important scattering processes for positrons are scatterings on electrons and on protons, whose cross section is the same as the one for electron (ee and ep) scattering, Eq. (1). As a consequence, Eq. (3) holds for $\bar{v}_{e}$ as well.

For the non-electron neutrinos, $v_{\mu}$ and $v_{\tau}$, and their antineutrinos, the physics of production is quite different. The number density of these neutrinos is determined at the number sphere. Outside this sphere neutrinos still scatter efficiently on leptons (until the energy sphere) and on nucleons (until the transport sphere) [42]. Hence, the WP size of non-electron neutrinos is determined by these scattering processes. In general, the size of the WP after a scattering process is determined by the time of overlap of the incoming WP and thus approximately equal to the size of the larger incoming WP [37]. As a consequence, neutrino WP will continue to broaden as long as they scatter with particles with larger WP sizes. In our case these particles are the leptons, since the nucleon WP are estimated to be very small; see Eq. (7). Con- 
sequently, we expect $\sigma_{x}$ for $v_{\mu}$ and $v_{\tau}$ to equal the electron WP size at the energy sphere. This means that once again the result is given by Eq. (3).

\subsection{Propagation decoherence}

Propagation of (flavor) mixed states is described by a system of the WP which correspond to the eigenstates of propagation, i.e., the eigenstates of the Hamiltonian in matter, $v_{i m}$ $(i=1,2,3){ }^{2}$ The group velocities of the eigenstates are determined by the eigenvalues of the Hamiltonian:

$v_{i m}=\frac{\mathrm{d} H_{i m}}{\mathrm{~d} p}$.

We will consider a two-neutrino system characterized by a mass squared difference $\Delta m^{2} \equiv m_{2}^{2}-m_{1}^{2}$ and a vacuum mixing angle $\theta$. Using the explicit expressions for $H_{i m}$ in the two-neutrino case, we find the difference of the eigenvalues

$\Delta H_{m} \equiv H_{2 m}-H_{1 m}=\frac{\Delta m^{2}}{2 E} R(\xi)$,

where

$R(\xi) \equiv \sqrt{(\cos 2 \theta-\xi)^{2}+\sin ^{2} 2 \theta}$

and $^{3}$

$\xi \equiv \frac{2 E V}{\Delta m^{2}}$

with the matter potential $V= \pm \sqrt{2} G_{F} n_{e}$ for neutrinos and antineutrinos, respectively. Here $G_{F}$ is the Fermi coupling constant. In vacuum $R=1$.

Due to the difference of the group velocities,

$\Delta v_{m} \equiv v_{1 m}-v_{2 m}=-\frac{\mathrm{d} \Delta H_{m}}{\mathrm{~d} p}$,

the WP shift with respect to each other in configuration space. After traveling a distance $L$ the shift equals

$\Delta x_{\text {shift }}=\int_{0}^{L} \mathrm{~d} x \Delta v_{m}$,

which is $\Delta v_{m} L$ for a medium with constant density. If the size of the WP does not change in the course of propagation, they cease to overlap when $\left|\Delta x_{\text {shift }}\right| \simeq \sigma_{x}$, which is called propagation decoherence. The distance $L_{\mathrm{coh}}$ at which this happens, the coherence length [44], is determined by

$\left|\int_{0}^{L_{\mathrm{coh}}} \mathrm{d} x \Delta v_{m}\right|=\sigma_{x}$

2 The eigenstates of the Hamiltonian should be considered the "true particles", in analogy to the concept of quasi-particles in condensed matter physics [43].

3 We use the variable $\xi$ instead of $\eta=1 / \xi$ in [28].
For constant density this gives

$L_{\mathrm{coh}}=\frac{\sigma_{x}}{\left|\Delta v_{m}\right|}$.

In vacuum Eqs. (13-15) are reduced to well-known results [19]: the eigenstates of the Hamiltonian are the mass eigenstates, $v_{i m}=v_{i}$, the difference of group velocities equals

$\Delta v=\frac{\Delta m^{2}}{2 E^{2}}$,

and the coherence length according to Eq. (15) is

$L_{\mathrm{coh}}=\sigma_{x} \frac{2 E^{2}}{\Delta m^{2}}$.

In the three-neutrino system there are three different coherent lengths which correspond to three difference modes of oscillations driven by three different $\Delta m_{i j}^{2} \equiv m_{i}^{2}-m_{j}^{2}$.

In matter with constant or slowly changing density, the difference of velocities of the eigenstates equals [28] according to (12) and (9)

$\Delta v_{m}=\frac{\Delta m^{2}}{2 E^{2}} \frac{1-\xi \cos 2 \theta}{\sqrt{1-2 \xi \cos 2 \theta+\xi^{2}}}=\frac{\Delta m^{2}}{2 E^{2}} \frac{1-\xi \cos 2 \theta}{R(\xi)}$.

Equation (18) gives $\Delta v_{m} \simeq \Delta v$ in the limit of small densities, $|\xi| \ll 1$, and

$\Delta v_{m} \simeq-\operatorname{sgn}(\xi) \frac{\Delta m^{2}}{2 E^{2}} \cos 2 \theta=-\operatorname{sgn}(\xi) \Delta v \cos 2 \theta$

in the matter-dominated case, $|\xi| \gg 1$. For small mixing we obtain again the vacuum value, up to a possible sign change. This is related to the fact that the matter potential $V$ does not depend on energy and therefore does not produce dispersion. Correspondingly, $L_{\mathrm{coh}}$ is close to the vacuum value everywhere apart from the resonance region in the resonance channel with $\xi>0$. This is realized for neutrinos and the normal mass hierarchy, and for antineutrinos and the inverted mass hierarchy. In the non-resonance channel, $\xi<0$ (i.e., for antineutrinos and a normal mass hierarchy, and for neutrinos and the inverted hierarchy), the difference of velocities and coherence length are close to the vacuum values everywhere.

In matter with density such that

$\xi=\xi_{0} \equiv \frac{1}{\cos 2 \theta}$,

$\Delta v_{m}=0$ [45], so the WP do not shift and separate. In this case $L_{\text {coh }} \rightarrow \infty .{ }^{4}$ We will call $\xi_{0}$ the critical value and the corresponding density and energy the critical density and critical energy. The critical value is larger than the resonance value $\xi_{R}=\cos 2 \theta$, as $\xi_{0}=\xi_{R} / \cos ^{2} 2 \theta$. For small mixing it is close to $\xi_{R}$.

\footnotetext{
4 The WP still spread; see Sect. 5.
} 
When neutrinos propagate from large to small densities, $\Delta v_{m}$ changes sign near the MSW resonance. This can lead to the interesting phenomenon that WP separate above (in density) the resonance, then approach each other below the resonance and overlap at some point, thus restoring coherence $[28,45]$. This can be realized for solar and supernova neutrinos.

In the case of quickly changing density (strong adiabaticity violation) the instantaneous eigenstates may become irrelevant for the description of the flavor evolution. So the WP and group velocities introduced for these eigenstates have limited (or no) sense.

\subsection{Wave packet separation and coherence loss} in a supernova

When propagating from the neutrinosphere to the surface of the star, neutrinos cross regions with changing conditions which affect propagation and coherence of WP. In the central parts, neutrino-neutrino scattering leads to the potential $V_{\nu \nu}$ $[2,4]$. This potential is much smaller than the usual matter potential $V$ in the neutrinosphere, but it can be comparable to $V$ or even bigger at distances of order $10^{2} \mathrm{~km}$ from the center during later phases of the supernova explosion. $V_{v v}$ depends on the neutrino flavor state (i.e., on the neutrino wave function), which leads to the so-called collective oscillations.

Outside the regions and time period where $V_{\nu \nu}$ is important we can use the results of the previous subsection. Above resonances the difference of group velocities is given by Eq. (19) and does not depend on density. So, the integration in Eq. (14) is trivial, and consequently we obtain for the coherence length

$L_{\mathrm{coh}} \simeq \sigma_{x} \frac{2 E^{2}}{\Delta m^{2} \cos 2 \theta}$.

Using the range for $\sigma_{x}$ from Eq. (4), this gives for the 13 oscillation mode driven by $\left|\Delta m_{31}^{2}\right| \simeq 2.5 \cdot 10^{-3} \mathrm{eV}^{2}$ and $\sin ^{2} \theta_{13} \simeq 0.022$ as well as for the 1-2 mode with $\Delta m_{21}^{2} \simeq$ $7.5 \cdot 10^{-5} \mathrm{eV}^{2}$ and $\sin ^{2} \theta_{12} \simeq 0.30$ [46]

$$
\begin{aligned}
& L_{\mathrm{coh}}^{(13)} \simeq(30-60) \mathrm{km}\left(\frac{E}{15 \mathrm{MeV}}\right)^{2}, \\
& L_{\mathrm{coh}}^{(12)} \simeq(2.1-4.5) \cdot 10^{3} \mathrm{~km}\left(\frac{E}{15 \mathrm{MeV}}\right)^{2} .
\end{aligned}
$$

The estimate (22) shows that the coherence length $L_{\mathrm{coh}}^{(13)}$ of supernova neutrinos is shorter than or similar to the distance to the region where flavor evolution starts, in particular where collective effects due to $v v$ scattering become operative. This motivates studying decoherence effects on collective oscillations.

\section{Decoherence and averaging over neutrino energy}

So far we have considered WP in configuration space. We have also assumed that the coherence length is determined entirely by the production process. Alternatively, we can consider decoherence in energy-momentum space, where the WP width is $\sigma_{E} \simeq \sigma_{p} \simeq 1 / \sigma_{x}$. This consideration makes it easier to take into account the detection process.

\subsection{Coherence in energy-momentum space}

We generalize here known results for propagation in vacuum. The oscillation phase is

$\phi(E, L)=\frac{\Delta m^{2} L}{2 E}$,

and the interference term in the oscillation probability is proportional to $\cos \phi$. This term leads to the characteristic oscillatory pattern that can be observed in experiments. The probability has to be averaged over the size of the WP in momentum space. This averaging suppresses the interference term if the spread of phases within the WP is larger than $2 \pi$. Consequently, the coherence length can be defined via the condition

$|\Delta \phi|=\left|\phi\left(E-\sigma_{E}, L_{\mathrm{coh}}\right)-\phi\left(E+\sigma_{E}, L_{\mathrm{coh}}\right)\right|=2 \pi$.

Using Eq. (24) we obtain

$L_{\mathrm{coh}}=\frac{2 \pi}{\sigma_{E}} \frac{E^{2}}{\left|\Delta m^{2}\right|}\left(1-\frac{\sigma_{E}^{2}}{E^{2}}\right) \simeq \frac{\pi}{\sigma_{E}} \frac{2 E^{2}}{\left|\Delta m^{2}\right|}$.

This result for the coherence length has the same form as Eq. (17) but is larger by a factor of $\pi$. Given the fact that the definitions (14) and (25) are ad hoc and do not take into account that coherence is not lost abruptly, it is not surprising that the results for $L_{\text {coh }}$ agree up to a factor of order one. In the rigorous quantum field theory treatment, no such discrepancy arises [33,37,39].

We can immediately generalize the discussion to the case of matter with adiabatically varying density. The oscillation phase is now

$\phi(E, L)=\int_{0}^{L} \mathrm{~d} x \Delta H_{m}$,

where $\Delta H_{m}$ is given in Eq. (9). Taylor-expanding $\phi$ in Eq. (25), which is justified as long as $\sigma_{E} / E \ll 1$, we obtain

$$
\begin{aligned}
\Delta \phi & \simeq-\left.2 \sigma_{E} \frac{\partial \phi}{\partial E}\right|_{E, L_{\mathrm{coh}}}=-2 \sigma_{E} \frac{\partial}{\partial E} \int_{0}^{L_{\mathrm{coh}}} \mathrm{d} x \Delta H_{m} \\
& =-2 \sigma_{E} \int_{0}^{L_{\mathrm{coh}}} \mathrm{d} x \frac{\mathrm{d}}{\mathrm{d} E} \Delta H_{m} .
\end{aligned}
$$


Then the condition $|\Delta \phi|=2 \pi$ gives

$\left|\int_{0}^{L_{\mathrm{coh}}} \mathrm{d} x \frac{\mathrm{d}}{\mathrm{d} E} \Delta H_{m}\right| \simeq \frac{\pi}{\sigma_{E}}$.

On the other hand, according to Eqs. (8) and (13), the separation of the two WP in configuration space is ${ }^{5}$

$\Delta x_{\text {shift }}=-\int_{0}^{L} \mathrm{~d} x \frac{\mathrm{d}}{\mathrm{d} E} \Delta H_{m}$.

Therefore the decoherence condition $\left|\Delta x_{\text {shift }}\right|=\sigma_{x}$ yields

$\left|\int_{0}^{L_{\mathrm{coh}}} \mathrm{d} x \frac{\mathrm{d}}{\mathrm{d} E} \Delta H_{m}\right|=\sigma_{x} \simeq \frac{1}{\sigma_{E}}$,

which is the same condition as Eq. (29), again up to a spurious factor of $\pi$.

The key point of this equivalence is that both the difference of group velocities of the mass eigenstates and the difference of the oscillation phases for different energies in the WP are determined by the same quantity $\mathrm{d}\left(\Delta H_{m}\right) / \mathrm{d} E$. According to Eqs. (28) and (30),

$\Delta x_{\text {shift }}=-\frac{\partial \phi}{\partial E}$,

which holds for arbitrary $L \neq L_{\mathrm{coh}}$, too.

A zero value of $\Delta x_{\text {shift }}$, i.e., no shift of the WP, corresponds to a zero derivative $\partial \phi / \partial E$. This implies a weak dependence of the oscillation phase on energy in a certain interval, consequently no significant effect of averaging, and thus no decoherence. However, higher-order terms in the Taylor expansion of the oscillation phase lead to $\Delta \phi \neq 0$ even if $\partial \phi / \partial E$ vanishes. Therefore, the consideration in momentum space implies that the coherence length increases significantly for $\partial \phi / \partial E=0$ but does not become infinite, which is different from what we observed using the configuration-space treatment in Sect. 2.2.

\subsection{Impact of the detection process}

In the adiabatic case considered so far, loss of WP overlap has no effect in practice, once the energy resolution of the detector $\Delta E$ is taken into account. Indeed, in order to observe the oscillation pattern, $\Delta E$ has to be sufficiently small to satisfy

$|\phi(E-\Delta E, L)-\phi(E+\Delta E, L)|<2 \pi$,

which is analogous to Eq. (25) with $\sigma_{E}$ replaced by $\Delta E$ and in fact suffices to guarantee the observation of oscillations even if Eq. (25) is violated. In configuration space a small energy uncertainty $\Delta E$ implies a large time uncertainty $\Delta t$. All WP arriving within this time interval will be detected coherently and hence their effects in the detector can interfere

\footnotetext{
${ }^{5}$ As neutrinos are highly relativistic, $\mathrm{d} / \mathrm{d} E \simeq \mathrm{d} / \mathrm{d} p$.
}

even if they do not overlap. In this way, the detector restores coherence [20].

If inequality (33) is violated, averaging the oscillation probability over the energy interval $[E-\Delta E, E+\Delta E]$ destroys the oscillation pattern just like the separation of the WP or averaging over the WP size in momentum space. As a short cut, we could take into account the detection process by introducing a generalized WP with width $\sigma_{E \text {,tot }}$ such that [33]

$\frac{1}{\sigma_{E, \text { tot }}^{2}}=\frac{1}{\sigma_{E}^{2}}+\frac{1}{\Delta E^{2}}$.

\subsection{Equivalence of wave packet separation and energy averaging}

Essentially, Eq. (25) means that $2 \sigma_{E}$ equals the period of the oscillatory pattern in energy when $L=L_{\mathrm{coh}}$, which we denote by $E_{T}\left(L_{\text {coh }}\right)$. Then from Eqs. (29) and (30) we obtain $\left|\Delta x_{\text {shift }}\right|=\pi / \sigma_{E}$ or

$E_{T}=\frac{2 \pi}{\left|\Delta x_{\text {shift }}\right|}$.

As one can easily verify, Eq. (35) holds for any baseline $L$. It is this relation between the period of the oscillatory curve in energy and the shift of the WP in configuration space that ensures equivalence of results obtained in the momentum and configuration-space considerations. One could say equally well that the equivalence is due to Eq. (32), without having to introduce $E_{T}$.

In the case of complete overlap, $\Delta x_{\text {shift }} \rightarrow 0$, Eq. (35) gives $E_{T} \rightarrow \infty$, which is equivalent to $\partial \phi / \partial E \rightarrow 0$. In this case the effect of the interference term (deviation from the averaged probability) does not depend on energy, and therefore it is also independent of the energy resolution of the detector (as long as $\Delta E \gg E$, as required by the Taylor expansion in Eq. (28)).

In the opposite case of large shift $\Delta x_{\text {shift }}$, the period becomes very small, so one needs to have very good energy resolution since the condition $\Delta E \ll E_{T}$ should be satisfied to observe the interference (oscillatory effect) in the oscillation probability. In configuration space that would correspond to a long coherent observation time with $\Delta t \sim 1 / \Delta E$, and consequently to restoration of coherence in the detector.

Summarizing the two pictures, in both representations (configuration and momentum space) we start from the eigenvalues of the Hamiltonian $H_{i m}$ and their difference $\Delta H_{m}$. In configuration space $\Delta H_{m}$ determines the difference of group velocities and the relative shift of the WP. Then comparing the shift with the effective size of the packet $\sigma_{E \text {,tot }}^{-1}$ (which includes both the produced WP and the energy resolution of the detector) determines whether coherence is preserved or lost. In momentum space $\Delta H_{m}$ determines the oscilla- 
tion phase and the oscillatory period in energy $E_{T}$. Comparison of the latter with the effective width of the packet $\sigma_{E \text {,tot }}$ determines whether the oscillatory pattern is observable or averaged to a constant oscillation probability.

Equivalence of the configuration- and momentum-space considerations is realized when the whole process is taken into account: production, propagation and detection of neutrinos. The phase $\phi(E, L)$ is the key (integral) characteristic which takes into account all the relevant (for coherence) features of propagation.

The discussion up to this point shows that WP separation and energy averaging produce equivalent effects in the adiabatic case. In fact, this also follows from theorems in [20,34], according to which it is impossible to distinguish long and short WP; in particular, whether one can observe coherent effects or not is independent of the size of the WP. In the following, we will consider neutrino oscillations in matter with density jumps, aiming to show explicitly that the equivalence holds under such conditions as well.

\section{Coherence in multi-layer medium}

The picture described in the previous section is modified if adiabaticity is broken. In the following we will consider special (maximal) adiabaticity breaking occurring when neutrinos propagate in a multi-layer medium that consists of several layers with constant or adiabatically changing density and abrupt density changes between the layers. In other words, there is a step-like change or jump of density at each border between two layers. This happens in a supernova at the front of the shock waves. Later, neutrinos experience density jumps when they enter the Earth and at the boundary between the mantle and the core. Coherence in such a case can be treated in the same way as before, taking into account splittings of the eigenstates at the borders.

\subsection{Splitting of eigenstates}

Let us consider the jump of the density between the layers $k$ and $k+1$. Suppose a neutrino propagates in the layer $k$, crosses the border and then propagates in the layer $k+1$. The eigenstate $v_{i m}^{(k)}$ in the layer $k$ does not coincide with any eigenstate in the layer $k+1$. Therefore, when crossing the border, $v_{i m}^{(k)}$ will split into two $0^{6}$ eigenstates $v_{j m}^{(k+1)}$ of layer $k+1$. Correspondingly, at a density jump each WP splits up into a pair of new packets. After the split, the state will oscillate and the packets will shift according to the group velocity difference in the second layer.

Crossing a medium with $n$ layers, $2^{n}$ components of the neutrino state are generated. They correspond to parts of the

\footnotetext{
${ }^{6}$ In the case of two-neutrino mixing.
}

WP of $v_{1 m}^{(n)}$ and $v_{2 m}^{(n)}$ with different shifts. Thus, compared to the adiabatic case where one deals with only two WP that either overlap or do not, there are additional possibilities in the multi-layer medium. It is possible that some but not all WP overlap in the detector, allowing to observe a part of the interference terms in the oscillation probability. This corresponds to an intermediate case between complete coherence (all WP overlap) and complete decoherence (no WP overlap). Notice that the splitting has sense only in the presence of shift and separation of the WP. If the shift is neglected in each layer we can sum up the components which belong to the same eigenstate and the picture is reduced again to the propagation of two WP.

The splitting of eigenstates at a density jump corresponds to the decomposition

$$
\left(\begin{array}{c}
v_{1 m}^{(k)} \\
v_{2 m}^{(k)}
\end{array}\right)=\left(\begin{array}{cc}
c_{k+1, k} & s_{k+1, k} \\
-s_{k+1, k} & c_{k+1, k}
\end{array}\right)\left(\begin{array}{c}
v_{1 m}^{(k+1)} \\
v_{2 m}^{(k+1)}
\end{array}\right),
$$

where

$c_{k+1, k} \equiv \cos \left(\theta_{k+1}-\theta_{k}\right), \quad s_{k+1, k} \equiv \sin \left(\theta_{k+1}-\theta_{k}\right)$

are the cosine and sine of the change of the mixing angle at the jump,

$\theta_{k+1, k} \equiv \theta_{k+1}-\theta_{k}$

and $\theta_{k}$ is the mixing angle in matter in the layer $k$. If the matter density varies adiabatically within the layers, we use $\theta_{k+1, k} \equiv \theta_{k+1}^{(\mathrm{i})}-\theta_{k}^{(\mathrm{f})}$, the difference of the mixing angle at the beginning of layer $k+1$ and the one at the end of layer $k$. The vacuum mixing angle is $\theta_{0} \equiv \theta$. We will also use $c_{k} \equiv \cos \theta_{k}$ and $s_{k} \equiv \sin \theta_{k}$.

For the difference of mixing angles we find

$\sin 2\left(\theta_{k+1}-\theta_{k}\right)=\frac{\xi_{k+1}-\xi_{k}}{R\left(\xi_{k+1}\right) R\left(\xi_{k}\right)} \sin 2 \theta$,

where $R(\xi)$ is defined in Eq. (10). In the case of small densities, $\left|\xi_{k}\right| \ll 1$, we have

$\sin 2\left(\theta_{k+1}-\theta_{k}\right) \simeq\left(\xi_{k+1}-\xi_{k}\right) \sin 2 \theta$.

\subsection{Two layers of matter and catch-up effect}

As a simple explicit example, let us consider vacuum followed by two layers of matter with lengths $L_{1}$ and $L_{2}$, constant densities $\rho_{1}$ and $\rho_{2}$ and the corresponding effective mixing angles $\theta_{1}$ and $\theta_{2}$. At the end of the second layer we place a detector sensitive to $v_{e}$. Suppose the mass eigenstate $v_{1}$ arrives at the border of the first layer. Here the state splits into a pair of new WP corresponding to the eigenstates in matter with density $\rho_{1}$,

$v_{1}=c_{10} v_{1 m}^{(1)}+s_{10} v_{2 m}^{(1)}$. 
The new eigenstates $v_{i}^{(1)}$ propagate to the end of the layer, acquiring the oscillation phase $\phi_{1}=\Delta H_{m}^{(1)} L_{1}$. The abrupt density change from $\rho_{1}$ to $\rho_{2}$ transforms the eigenstates in matter according to Eq. (36) with $k=1$. Before reaching the detector they acquire another oscillation phase $\phi_{2}=\Delta H_{m}^{(2)} L_{2}$.

Assuming complete coherence, the probability for observing an electron neutrino in the detector is then (for a single neutrino energy $E)^{7}$

$$
\begin{aligned}
P_{\nu_{1} \rightarrow v_{e}}(E)= & \mid c_{2} c_{21} c_{10}-c_{2} s_{21} s_{10} e^{i \phi_{1}}+s_{2} s_{21} c_{10} e^{i \phi_{2}} \\
& +\left.s_{2} c_{21} s_{10} e^{i\left(\phi_{1}+\phi_{2}\right)}\right|^{2} .
\end{aligned}
$$

Here we have projected the eigenstates in the second layer onto $v_{e}$ according to $v_{e}=c_{2} v_{1 m}^{(2)}+s_{2} v_{2 m}^{(2)}$. The four terms in Eq. (42) correspond to the four components of the state after two splits. The interference terms are proportional to $\cos \phi_{1}$, $\cos \phi_{2}, \cos \left(\phi_{1}+\phi_{2}\right)$, and $\cos \left(\phi_{1}-\phi_{2}\right)$, that is, to the cosines of all possible combinations of the two phases.

The splitting of WP at each boundary can lead to the particularly interesting situation that although no WP overlap for some time during the propagation, two WP overlap again when they reach the detector. Suppose that $v_{1 m}^{(k)}>v_{2 m}^{(k)}$ in both layers. Then $v_{2 m}^{(1)}$ falls behind during the propagation through the first layer. However, its splitting at the boundary creates a $v_{1 m}^{(2)}$ WP, which can catch up with the $v_{2 m}^{(2)}$ WP that originated from $v_{1 m}^{(1)}$, as illustrated in Fig. 1. These two WP overlap in the detector, independently of their size, if

$\Delta v_{m}^{(1)} L_{1}=\Delta v_{m}^{(2)} L_{2}$,

where $\Delta v_{m}^{(k)}$ denotes the group velocity difference in layer $k$. Hence, the observed oscillation probability $P_{\nu_{1} \rightarrow v_{e}}$ will contain the corresponding interference term. As $\Delta v_{m}^{(k)}$ depends on energy, the catch-up condition Eq. (43) can only be satisfied for a particular energy, however.

This catch-up effect depends crucially on the WP picture in configuration space. At first sight, it seems unlikely to recover such a complicated effect in the momentum-space picture. Thus, one might hope that this effect breaks the equivalence between WP separation and energy averaging, potentially allowing to construct a setup where the observed oscillation probability depends on the WP size. This is not the case, though. According to Eq. (32),

$\Delta v_{m}^{(k)} L_{k}=\Delta x_{\text {shift }}^{(k)}=-\frac{\partial \phi_{k}}{\partial E}$.

Therefore, Eq. (43) is equivalent to

$\frac{\partial}{\partial E}\left(\phi_{1}-\phi_{2}\right)=0$.

7 The oscillation probability and the phases depend not only on $E$ but also on $L_{k}$ and $\rho_{k}$, but we do not write these dependences explicitly in the following.

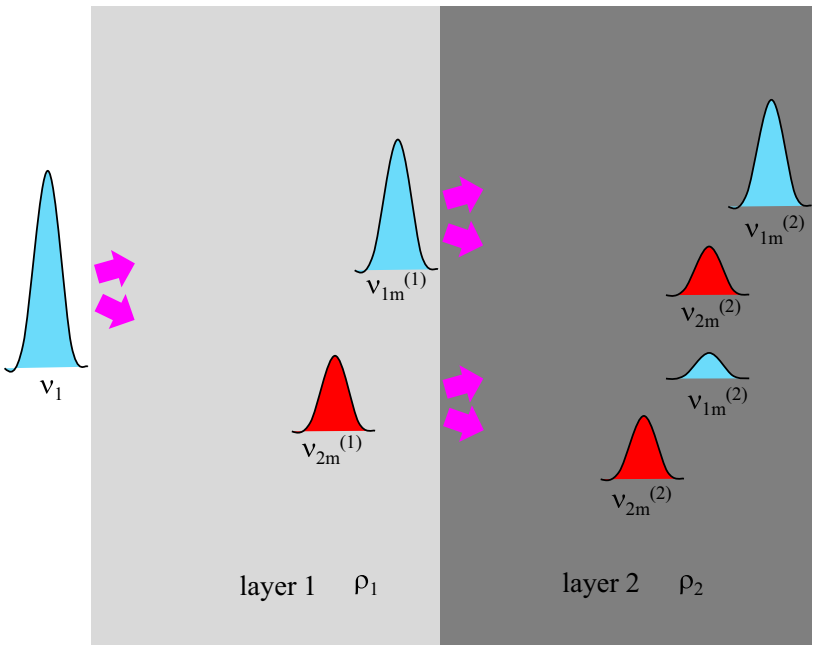

Fig. 1 Illustration of the splitting of the neutrino states and catch-up effect in a medium with two layers. The wave packet $v_{1 m}^{(2)}$ catches up with the packet $v_{2 m}^{(2)}$, so that they start to overlap in the second layer

This condition implies that one combination of oscillation phases, $\phi_{1}-\phi_{2}$, depends only weakly on energy. Then averaging $P_{v_{1} \rightarrow v_{e}}(E)$ over $\sigma_{E}$ or $\Delta E$ will not suppress the interference term proportional to $\cos \left(\phi_{1}-\phi_{2}\right)$. Consequently, the observed oscillation probability will contain this term. Once again, the considerations in configuration and momentum space lead to the same result.

Let us study energy averaging more systematically in order to find how much the coherence length can grow due to the catch-up effect and to interpret the remaining combinations of oscillation phases as well. The experimentally observed oscillation probability is given by the energy average

$\overline{P(E)}=\int \mathrm{d} E^{\prime} P\left(E^{\prime}\right) f\left(E^{\prime}\right)$,

where $f(E)$ is the energy resolution function of the detector. As a first approximation, we can replace $f(E)$ by a step function that is non-zero only in the interval $[E-\Delta E, E+$ $\Delta E]$, where $\Delta E$ is the energy resolution.

Let us consider one combination of oscillation phases and denote it by $\psi(E)$. Analogously to Eq. (33), $\cos \psi$ in the corresponding interference term survives energy averaging as long as

$|\Delta \psi(E)|=|\psi(E-\Delta E)-\psi(E+\Delta E)|<2 \pi$.

The Taylor expansion of $\psi$ yields

$\Delta \psi(E)=-\left.2 E \frac{\partial \psi}{\partial E}\right|_{E} \frac{\Delta E}{E}-\left.\frac{E^{3}}{3} \frac{\partial^{3} \psi}{\partial E^{3}}\right|_{E}\left(\frac{\Delta E}{E}\right)^{3}+\cdots$ 
Thus, the variation of $\psi$ around the energy satisfying

$$
\left.\frac{\partial \psi}{\partial E}\right|_{E}=0
$$

is suppressed by two additional powers of $\Delta E / E$ compared to the usual case. Correspondingly, the propagation length can be larger before $\Delta \psi$ reaches $2 \pi .^{8}$ In other words, the individual coherence length for the $\cos \psi$ interference term increases considerably, although it does not become infinite. In an experiment, this would show up as a deviation from the averaged oscillation probability expected in the totally incoherent case for a certain energy range and baseline. One would not observe a complete oscillation pattern in energy, since Eq. (49) can only be satisfied for a particular energy.

Next, we consider the stationarity condition (49) for different interference terms, i.e., different combinations $\psi$.

1. For $\psi=\phi_{k}(k=1,2)$, it can be satisfied only if $\xi_{k}=$ $\xi_{0}=(\cos 2 \theta)^{-1}$ and thus $\Delta v_{m}^{(k)}=0$, i.e., the WP do not separate in layer $k$. For this case we had found the stationary-phase condition already at the end of Sect. 3.1.

2. For $\psi=\phi_{1}-\phi_{2}$, we obtain Eq. (43), as already discussed. Using (18), we can write this equation as

$$
L_{2}=L_{1} \frac{1-\xi_{1} \cos 2 \theta}{1-\xi_{2} \cos 2 \theta} \frac{R\left(\xi_{2}\right)}{R\left(\xi_{1}\right)}
$$

As $L_{1}$ and $L_{2}$ are positive, this condition can only be satisfied if $\xi_{1}, \xi_{2}<(\cos 2 \theta)^{-1}$, or if $\xi_{1}, \xi_{2}>(\cos 2 \theta)^{-1}$, that is, if both densities are below or above the critical density. ${ }^{9}$ This means that either in both layers the eigenstates $v_{1 m}$ move faster than $v_{2 m}$, or in both layers $v_{1 m}$ move more slowly than $v_{2 m}$. The overlap occurs in the second layer between the WP of $v_{2 m}^{(2)}$ and $v_{1 m}^{(2)}$ originating from the transitions

$v_{1} \rightarrow v_{1 m}^{(1)} \rightarrow v_{2 m}^{(2)}$ and $\quad v_{1} \rightarrow v_{2 m}^{(1)} \rightarrow v_{1 m}^{(2)}$

where the arrows indicate the transitions at the density jumps. The corresponding interference term that is not averaged to zero arises from the second and third terms in the oscillation probability (42). According to Eq. (42) the oscillation depth for this mode is $4 s_{2} c_{2} s_{10} c_{10} s_{21}^{2}=$ $\sin 2 \theta_{2} \sin 2 \theta_{10} s_{21}^{2}$.

\footnotetext{
$\overline{8}$ Alternatively, for a fixed baseline a larger $\Delta E$ is sufficient to observe the interference term.

9 Condition (50) can also be satisfied for arbitrary densities in the nonresonance channel, where $\xi_{k}<0$.
}

3. For $\psi=\phi_{1}+\phi_{2}$, we obtain from Eq. (49) using Eqs. (44) and (18)

$$
L_{2}=-L_{1} \frac{\Delta v_{m}^{(1)}}{\Delta v_{m}^{(2)}}=-L_{1} \frac{1-\xi_{1} \cos 2 \theta}{1-\xi_{2} \cos 2 \theta} \frac{R\left(\xi_{2}\right)}{R\left(\xi_{1}\right)} .
$$

This condition can be satisfied if $\xi_{2}>(\cos 2 \theta)^{-1}>\xi_{1}$, or if $\xi_{1}>(\cos 2 \theta)^{-1}>\xi_{2}$, that is, if one density is larger than the critical density and the other one is smaller. Now either $v_{1 m}^{(1)}$ moves faster than $v_{2 m}^{(1)}$ whereas $v_{1 m}^{(2)}$ moves more slowly than $v_{2 m}^{(2)}$, or vice versa. The overlapping WP originate from the transitions

$$
v_{1} \rightarrow v_{1 m}^{(1)} \rightarrow v_{1 m}^{(2)} \text { and } \quad v_{1} \rightarrow v_{2 m}^{(1)} \rightarrow v_{2 m}^{(2)}
$$

In the limit of large densities, $\left|\xi_{k}\right| \gg 1$, the condition (50) reduces to

$L_{2} \simeq L_{1}\left[1-\frac{\sin ^{2} 2 \theta}{\cos 2 \theta}\left(\frac{1}{\xi_{1}}-\frac{1}{\xi_{2}}\right)\right]$.

For small densities, $\left|\xi_{k}\right| \ll 1$, we obtain

$L_{2} \simeq L_{1}\left[1-\frac{\sin ^{2} 2 \theta}{2}\left(\xi_{1}^{2}-\xi_{2}^{2}\right)\right]$.

So $L_{2} \simeq L_{1}$ in both limits. In addition, for small densities the corrections are proportional to the square of the small parameters $\xi_{k}$, i.e., strongly suppressed.

The case of two layers of matter with adiabatically varying density and density jump between them can be realized in a supernova. The jump is due to the shock wave. The new eigenstates propagate adiabatically and encounter the shock wave in the MSW transition region, where the change of mixing angle is large (the effect of the shock wave outside this region is very small). The first layer is between inner parts of the collective effects and the shock front, and the second one is between the shock front and the surface of the star. The oscillation phases $\phi_{k}$ should be computed for adiabatically varying density, $\phi_{k}=\int_{k} \mathrm{~d} x \Delta H_{m}^{(k)}$.

The possibility of two layers and two jumps is realized when there are two shock wave fronts (one can move inward); see, e.g., [47]. In this case the first layer is the one between the shock fronts and the second one is above the outer shock. The result is described by Eq. (42) with similar correspondence as in the first case.

\subsection{Generalization}

The analysis can be immediately generalized to the case of $n$ layers of matter. In this case $n$ phases $\phi_{k}$ appear, and the amplitude for flavor transitions can be written as

$\mathcal{A}(E)=\sum_{r} a_{r} e^{i \psi_{r}}$, 
where $\psi_{r}$ is any possible sum of the oscillation phases $\phi_{k}$ acquired in the individual layers, $\psi_{r}=\left\{0, \phi_{1}, \phi_{2}, \ldots, \phi_{n}\right.$, $\left.\phi_{1}+\phi_{2}, \ldots, \phi_{1}+\phi_{n}, \ldots, \phi_{1}+\phi_{2}+\phi_{3}, \ldots\right\}$, and $a_{r}$ are real numbers depending on energy only through the energy dependence of the mixing angles in matter. The number of phase combinations is $2^{n}$. If we start from a single mass eigenstate, the number of terms in Eq. (54) is also $2^{n}$.

The oscillation probability for a single energy is

$P(E)=|\mathcal{A}(E)|^{2}$.

For two layers and observation of $v_{e}$ it is given explicitly in Eq. (42). The probability contains interference terms depending on the cosines of all possible combinations of the phases. An experiment measures the energy-averaged probability $\overline{P(E)}$ defined in Eq. (46). We can distinguish three limiting cases. ${ }^{10}$

1. If the detector's energy resolution is good enough to resolve all interference terms,

$$
\overline{P(E)} \simeq P_{\mathrm{coh}}=|\mathcal{A}(E)|^{2}
$$

Coherence is completely preserved. As discussed in Sect. 3.2, it does not matter whether this happens because all WP overlap in the detector or because the detector restores coherence.

2. For a bad resolution, the oscillation probability is given by the incoherent sum

$$
\overline{P(E)} \simeq P_{\mathrm{decoh}}=\sum_{r} a_{r}^{2}
$$

Coherence is completely lost. All WP are separated (and the detector does not restore coherence), or averaging due to the bad energy resolution removes even interference terms corresponding to overlapping WP.

3. It is possible that some but not all interference terms in the probability survive averaging, for instance,

$\overline{P(E)} \simeq \sum_{r} a_{r}^{2}+2 a_{s} a_{t} \cos \left(\psi_{s}-\psi_{t}\right)$

We will refer to this case as partial survival of coherence. Survival of coherence is possible for each interference term. Therefore, we have to introduce an individual coherence length for each term.

In the configuration-space picture, there are different reasons for partial survival of coherence, depending on which

\footnotetext{
10 To simplify the discussion we assume that there is a sharp transition from coherence to decoherence, i.e., interference terms are either present in $\overline{P(E)}$ or disappear completely. Thus, we neglect that interference terms are suppressed but non-zero for a partial overlap of WP.
}

type of interference term survives. On the one hand, if it contains a combination of several phases, the survival is a consequence of the catch-up effect discussed in the previous subsection. In this case we can also speak of partial restoration of coherence, since the corresponding WP may cease to overlap for some time before the catch-up effect restores the overlap. The survival of terms with more than two phases corresponds to WP that have different speeds in more than two layers before they meet again. For more than two layers, it is possible that the catch-up effect causes more than two WP to overlap in the detector. In such a case, partial restoration of coherence occurs for several interference terms at the same time.

On the other hand, if the term containing $\cos \phi_{k}$ survives, this can be due to a vanishing velocity difference in one layer, $\Delta v_{m}^{(k)}=0$, or due to the detector restoring the coherence of consecutive WP that were separated in layer $k$. Of course, there is also the trivial possibility that one or more layers are so thin that WP do not separate inside them. Another trivial example of partial survival of coherence is realized in the case of three-neutrino mixing in a single layer when at large enough distances the oscillation modes due to $\Delta m_{31}^{2}$ and $\Delta m_{32}^{2}$ are averaged to zero, whereas the mode due to the small splitting $\Delta m_{21}^{2}$ is not.

Note that the catch-up effect described here relies on two ingredients, matter effects and strong (maximal) adiabaticity violation. It is thus different from the increase of the coherence length that is possible if $\Delta v_{m}$ changes sign during adiabatic propagation $[28,45]$. In that case, there is no splitting of the WP into many components, and only two WP arrive at the detector.

\section{Spread of the wave packets}

\subsection{Separation and spread}

In the course of propagation between a supernova and the Earth in vacuum, two effects occur: (1) separation of the WP and (2) spread (deliquescence) of each WP. Indeed, variations of the group velocity $v=p / E$, where $E=\sqrt{p^{2}+m^{2}}$, can be written as

$\Delta v=\frac{\partial v}{\partial\left(m^{2}\right)} \Delta m^{2}+\frac{\partial v}{\partial p} \Delta p=-\frac{p}{2 E^{3}} \Delta m^{2}+\frac{m^{2}}{E^{3}} \Delta p$.

Here the first term is the difference of the group velocities of the mass eigenstates as given in Eq. (16). ${ }^{11}$ This term is responsible for the relative shift and eventual separation of the WP of the mass eigenstates. The second term in Eq. (59) is the velocity dispersion due to different momenta in the WP. It is responsible for a spread of the WP of an individual

\footnotetext{
${ }^{11}$ Up to a sign arising from our definition of $\Delta v_{m}$, see Eq. (12).
} 
mass eigenstate. For $\Delta p=\sigma_{E}$ we obtain the width of the packet after traveling a distance $L$,

$\sigma_{\text {spread }}=\frac{m^{2}}{E^{3}} \sigma_{E} L$.

The spread depends on the absolute value of the mass, and for $\sigma_{E}=1 \mathrm{MeV}$ we obtain

$\sigma_{\text {spread }} \simeq 7 \mathrm{~m}\left(\frac{L}{10 \mathrm{kpc}}\right)\left(\frac{15 \mathrm{MeV}}{E}\right)^{3}\left(\frac{m^{2}}{7.5 \cdot 10^{-5} \mathrm{eV}^{2}}\right)$.

Thus, the WP of the two heavier states arriving at the Earth have a macroscopic size. For the lighest state, the size can be microscopic.

The separation of two mass eigenstates at the distance $L$ between a supernova and the Earth equals according to Eq. (16)

$\Delta x_{\text {shift }} \simeq 51 \mathrm{~m}\left(\frac{L}{10 \mathrm{kpc}}\right)\left(\frac{15 \mathrm{MeV}}{E}\right)^{2}\left(\frac{\Delta m^{2}}{7.5 \cdot 10^{-5} \mathrm{eV}^{2}}\right)$.

The ratio of spread and separation does not depend on distance and is given by

$\frac{\sigma_{\text {spread }}}{\Delta x_{\text {shift }}}=2\left(\frac{m^{2}}{\Delta m^{2}}\right)\left(\frac{\sigma_{E}}{E}\right)$.

It is determined by the relative difference of momenta and masses. For a hierarchical mass spectrum $\left(m^{2} \simeq \Delta m^{2}\right.$ for the heavier mass eigenstate) and $\sigma_{E} / E<1 / 2$, the separation is larger than the spread. A mild degeneracy with $m_{2}^{2} \gtrsim 8 \Delta m_{21}^{2}$ or an inverted mass hierarchy are sufficient to obtain $\Delta x_{\text {shift }}<\sigma_{\text {spread }}$ for $E=15 \mathrm{MeV}$ and $\sigma_{E}=1 \mathrm{MeV}$. In the case of an inverted hierarchy we have $m_{2}^{2} / \Delta m_{21}^{2} \simeq \Delta m_{31}^{2} / \Delta m_{21}^{2} \simeq 33$, so $\sigma_{\text {spread }} / \Delta x_{\text {shift }} \simeq 4$ and the WP of the eigenstates separated by $\Delta m_{21}^{2}$ will never cease to overlap. As we will show, in spite of this overlap the condition for coherence is not changed.

\subsection{Spread and energy redistribution}

The spread is related to a certain energy redistribution within the packet in configuration space: $E$ becomes a function of coordinate, with the highest energies in the front of the WP and the lowest energies in the back. This can be seen by dividing the original momentum range into $n$ small intervals with $p_{j+1}-p_{j}=\delta p=\sigma_{E} / n$. The whole WP with average momentum $\bar{p}$,

$\Psi(x, t)=\sum_{j} \int_{p_{j}}^{p_{j+1}} \frac{\mathrm{d} p}{(2 \pi)^{1 / 2}} f(p-\bar{p}) e^{i p x-i E t}$,

is then given by a sum over $n \mathrm{WP}$, which we will call the small $W P$. The momentum-space width of a small WP equals $\sigma_{E} / n$ and its spatial size is $n / \sigma_{E} \sim n \sigma_{x}$. Introducing the average momentum and energy in each interval, $\bar{p}_{j}$ and $\bar{E}_{j}$, the group velocity of the small WP is $v_{j}=\bar{p}_{j} / \bar{E}_{j}$. We choose $\delta p$ small enough to be able to neglect the spread of the small WP against their original size for a given baseline $L$. The bigger the baseline, the smaller $\delta p$ has to be taken.

The higher the $\bar{p}_{j}$ of a given small WP, the larger the group velocity $v_{j}$. The distance which such a WP propagates during time interval $t$ equals

$x_{j} \simeq\left(1-\frac{m^{2}}{2 \bar{p}_{j}^{2}}\right) t$.

According to this, the small WP with higher $\bar{p}_{j}$ will be in front of those with smaller $\bar{p}_{j}$. Therefore, in the whole WP the front (forward edge) will have the highest energy. We denote the corresponding average momentum by $\bar{p}_{n}$.

The dependence of the average momentum $\bar{p}_{j}$ on the distance from the front edge of the whole WP, $\Delta x_{j}$, can be found in the following way. After propagation during the time $t$, the position $x_{n}$ of the center of the small (front) WP with average momentum $\bar{p}_{n}$ is given by Eq. (65) with $j=n$. Consequently, the distance (shift) between the packets $j$ and $n$ equals

$$
\begin{aligned}
\Delta x_{j} & \equiv x_{n}-x_{j} \simeq \frac{m^{2} t}{2}\left(\frac{1}{\bar{p}_{j}^{2}}-\frac{1}{\bar{p}_{n}^{2}}\right) \\
& \simeq \frac{m^{2} L}{2} \frac{\Delta p_{j}\left(\bar{p}_{n}+\bar{p}_{j}\right)}{\bar{p}_{j}^{2} \bar{p}_{n}^{2}}
\end{aligned}
$$

where $\Delta p_{j} \equiv \bar{p}_{n}-\bar{p}_{j}$ and $L \simeq t$ is the baseline, the distance that the whole WP has traveled. Approximating $\bar{p}_{n} \simeq \bar{p}_{j} \simeq$ $\bar{p}$ in the sum and product of momenta, which is justified for $\sigma_{E} \ll \bar{p}$, we can rewrite Eq. (66) as

$\Delta x_{j} \simeq \Delta p_{j} L \frac{m^{2}}{\bar{p}^{3}}$.

In particular, for $j=1$ we obtain the spatial size of the whole WP, leading to $\sigma_{\text {spread }} \simeq \sigma_{E} L m^{2} / \bar{p}^{3}$, which reproduces the result in Eq. (60). This confirms the validity of the presented picture. Thus, the spread of the whole WP can be described as separation of the small WP in configuration space. Using Eq. (60) we can express Eq. (67) as

$\sigma_{\text {spread }} \Delta p_{j} \simeq \Delta x_{j} \sigma_{E}$

We will show now that it is this energy redistribution which keeps the coherence condition unchanged. The phase difference between the WP of two mass eigenstates (the oscillation phase) can be written as [26]

$\phi=(x-v t) \Delta p-\frac{\Delta m^{2}}{2 E} t$

In the case of packets without spread the difference of the average momenta equals $\Delta p \simeq \Delta m^{2} / 2 E$ and $x-v t \lesssim \sigma_{x}$. 


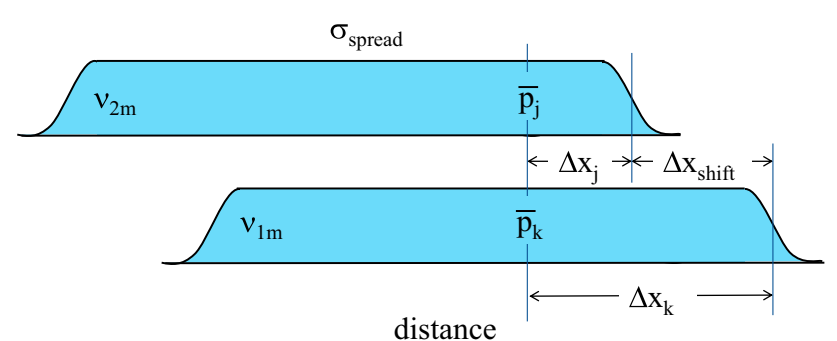

Fig. 2 Illustration of the shift of two wave packets with large spread. Loss of coherence occurs even if the packets overlap due to the spatial energy redistribution within the whole wave packets

Consequently, the first term in Eq. (69) is usually small. Here we consider the case with large spread of the WP instead, when $x-v t$ can be much larger than $\sigma_{x}$. Suppose we have two WP with nearly the same spread. This is realized, for example, for a mass eigenstate that spreads significantly on the way from a supernova to the Earth and splits into two eigenstates in matter upon entering the Earth; these two eigenstates then shift with respect to each other while propagating in the Earth. The additional spread during the propagation inside the Earth is negligible.

In order to calculate the oscillation probability we have to find the phase difference of the two WP in the same spacetime point. We split both WP into small WP as before. Let us consider the space-time point where the small WP with average momenta $\bar{p}_{j}$ and $\bar{p}_{k}$ are centered (the small WP with $\bar{p}_{j}$ is a part of the slower WP, while the other small WP with $\bar{p}_{k}$ belongs to the faster WP), as illustrated in Fig. 2. Let us find the difference between $\bar{p}_{j}$ and $\bar{p}_{k}$ arising from the spatial shift of the WP. According to Eq. (68),

$\Delta p_{j}=\Delta x_{j} \frac{\sigma_{E}}{\sigma_{\text {spread }}}, \quad \Delta p_{k}=\Delta x_{k} \frac{\sigma_{E}}{\sigma_{\text {spread }}}$.

As $\Delta x_{j}$ and $\Delta x_{k}$ are the distances from the front parts of the packets,

$\Delta x_{k}=\Delta x_{j}+\Delta x_{\text {shift }}$.

Using Eqs. (70) and (71) we obtain

$\Delta p_{j k} \equiv \bar{p}_{j}-\bar{p}_{k}=\Delta p_{k}-\Delta p_{j}=\frac{\sigma_{E}}{\sigma_{\text {spread }}} \Delta x_{\text {shift }}$.

This difference does not depend on $j$ and $k$. That is, the difference of momenta in the same space-time point is unchanged along the whole packets: $\Delta p=\Delta p_{j k}$.

Now $x-v t \simeq \pm \sigma_{\text {spread }}$ at the head and the tail of the WP, and therefore neglecting the last term in Eq. (69) we obtain

$\Delta \phi=\phi\left(x_{n}\right)-\phi\left(x_{1}\right) \simeq 2 \sigma_{\text {spread }} \Delta p=2 \sigma_{E} \Delta x_{\text {shift }}$.

Notice that $\sigma_{\text {spread }}$ cancels, which is related to the fact that a bigger spread implies a smaller gradient of $p$ and therefore a smaller $\Delta p$. The condition for loss of coherence, $|\Delta \phi|=2 \pi$, gives according to Eq. (73)
$\left|\Delta x_{\text {shift }}\right| \sigma_{E} \simeq \pi$

Remarkably, this coherence-loss condition for packets with spread coincides with the condition (29) for short packets without spread. This coincides with the conclusion we arrived at in energy-momentum space, thus confirming again the equivalence of the two considerations. As a consequence, we can neglect WP spread when discussing the coherence of supernova neutrinos oscillating in the Earth.

The coherence-loss condition (74) does not depend on the absolute neutrino mass scale, since $\sigma_{\text {spread }}$ cancels. It depends on $\Delta m^{2}$ via $\Delta x_{\text {shift }}$.

\subsection{Spread of the wave packets in matter}

Let us consider the spread of WP in matter, generalizing the result (60). In general, according to Eq. (8)

$\sigma_{\text {spread }}=\int_{0}^{L} \mathrm{~d} x \frac{\mathrm{d} v_{i m}}{\mathrm{~d} p} \Delta p=\int_{0}^{L} \mathrm{~d} x \frac{\mathrm{d}^{2} H_{i m}}{\mathrm{~d} p^{2}} \sigma_{E}$,

and the group velocities can be written as [28]

$v_{i m}=1-\frac{m_{1}^{2}+m_{2}^{2}}{4 p^{2}} \mp \frac{1}{2} \Delta v_{m}$,

where the upper sign ( - ) corresponds to $i=2$ and the lower one (+) to $i=1$. The difference of group velocities in matter, $\Delta v_{m}$, is given in Eq. (18). Differentiating with respect to $p$ and considering a constant matter density for simplicity, we obtain

$$
\begin{aligned}
\sigma_{i, \text { spread }}= & \frac{\sigma_{E} L}{2 p^{3}}\left[m_{1}^{2}+m_{2}^{2} \pm \Delta m^{2}\right. \\
& \left.\times \frac{1-3 \xi \cos 2 \theta+\frac{3}{2} \xi^{2}\left(1+\cos ^{2} 2 \theta\right)-\xi^{3} \cos 2 \theta}{\left(1-2 \xi \cos 2 \theta+\xi^{2}\right)^{3 / 2}}\right] .
\end{aligned}
$$

In the limit of small densities, $|\xi| \ll 1$, this expression reproduces the vacuum result (60). In the opposite case of large densities, $|\xi| \gg 1$, Eq. (77) gives

$\sigma_{i, \text { spread }} \simeq \frac{\sigma_{E} L}{2 p^{3}}\left(m_{1}^{2}+m_{2}^{2} \mp \operatorname{sgn}(\xi) \Delta m^{2} \cos 2 \theta\right)$.

In the case of large mixing, even if the spectrum is hierarchical the spread of both packets will be comparable and given by the larger mass.

The most interesting situation is the resonance region. For the critical value $\xi=1 / \cos 2 \theta$, which corresponds to equal group velocities, $\Delta v_{m}=0$, we obtain

$\sigma_{i, \text { spread }}=\frac{\sigma_{E} L}{2 p^{3}}\left(m_{1}^{2}+m_{2}^{2} \pm \frac{\Delta m^{2}}{2 \tan 2 \theta}\right)$.

This can be rewritten in general as

$\sigma_{i, \text { spread }}=\frac{\sigma_{E} L}{2 p^{3}}\left[(1 \mp a) m_{1}^{2}+(1 \pm a) m_{2}^{2}\right]$. 
If $a>1$, then for one of the WP we can obtain $\sigma_{i \text {,spread }}<$ 0 , which means that the WP shortens because its components with smaller energies move faster than the high-energy components. In the case of a normal mass hierarchy, $m_{2}^{2} \gg m_{1}^{2}$, this happens for the WP of the lighter mass eigenstate, for which $\sigma_{1 \text {, spread }} \simeq-\left(\sigma_{E} L / 2 p^{3}\right)(a-1) m_{2}^{2}$. If the density varies on the way of the neutrinos, this may lead to the interesting phenomenon that one of the packets first spreads and then shortens again. In the specific case of Eq. (79),

$a=\frac{1}{2 \tan 2 \theta}$,

and $a \simeq 1.6$ for the $1-3$ mixing, whereas $a<1$ for the $1-2$ mixing.

In the MSW resonance $\xi=\cos 2 \theta$, so

$\sigma_{i, \text { spread }}=\frac{\sigma_{E} L}{2 p^{3}}\left(m_{1}^{2}+m_{2}^{2} \pm \Delta m^{2} \frac{1+\sin ^{2} 2 \theta}{2 \sin 2 \theta}\right)$.

Now

$a=\frac{1+\sin ^{2} 2 \theta}{2 \sin 2 \theta} \simeq 1.9$

for the 1-3 mixing. For the 1-2 mixing, the parameter $a$ is only slightly bigger than 1 .

In the realistic situation of a supernova this may happen in the MSW region. However, most of the spread occurs in vacuum on the way to the Earth, and the spread inside the supernova can be neglected.

\section{Oscillations of supernova neutrinos inside the Earth}

\subsection{Neutrino states at the surface of the Earth}

On the way from a supernova to the Earth the coherence of mass eigenstates is lost. In configuration space the WP are separated by a macroscopic distance given by Eq. (62). Due to spread, the size of the WP, $\sigma_{\text {spread }} \sim$ few meters, is also macroscopic, but this does not prevent decoherence, as shown in Sect. 5.2. Furthermore, the coherence cannot be restored by the detector. Being separated by, say, $30 \mathrm{~m}$ the WPs arrive at the detector within a time interval of $10^{-6} \mathrm{~s}$. So the whole detector would have to be in a coherent state during $t>10^{-6} \mathrm{~s}$. This can be clearly seen in the energy representation. To restore coherence the energy resolution of the detector should be smaller than the period of oscillations in energy, $E_{T}$. The latter is determined by the condition $|d \phi / d E| E_{T}=2 \pi$, which is similar to the coherence condition (25) considered before. Taking the expression for the phase in vacuum we obtain from this equality

$E_{T}=E \frac{l_{v}}{L}=2.5 \cdot 10^{-15} E$, where $l_{v}=4 \pi E / \Delta m^{2}$ is the oscillation length. A resolution $\Delta E / E<10^{-15}$ cannot be obtained. Thus, each WP of a mass eigenstate will evolve inside the Earth independently and the results of their interactions in a detector will sum up incoherently.

As we have discussed in Sect. 4, entering the Earth each mass eigenstate splits into eigenstates $v_{i m}$ in the matter of the Earth and oscillates. We neglect the presence of the third neutrino $v_{3 m} \simeq v_{3}$ here. This state decouples from the rest of the system producing just a small (given by $\sin ^{2} \theta_{13}$ ) average oscillation result. So we will consider two-neutrino oscillations driven by the mass splitting $\Delta m_{21}^{2}$.

The Earth matter density is relatively small, yielding for the difference of group velocities

$\left|\Delta v_{m}\right| \simeq \frac{\Delta m_{21}^{2}}{2 E^{2}} K$

where $K \simeq 1$ for $\xi \ll 1$, which is realized at low energies. Using Eq. (18) we obtain $K=0.995$ and 0.985 for $E=$ $30 \mathrm{MeV}$ and $50 \mathrm{MeV}$, respectively. For the core and $E=$ $50 \mathrm{MeV}$ we find $\xi=0.35$ and $K=0.932$. So, even for relatively large $\xi$ the parameter $K$ is close to unity.

\subsection{The coherence condition in the Earth}

The Earth is a low-density medium for supernova neutrinos with $E<30 \mathrm{MeV}$. Then, using the vacuum value for the difference of group velocities (as a first approximation) we find for the shift (separation) of the eigenstates in the Earth

$$
\begin{aligned}
\Delta x_{\text {shift }} & \simeq L_{E} \Delta v=L_{E} \frac{\Delta m^{2}}{2 E^{2}} \\
& =3.3 \cdot 10^{-10} \mathrm{~cm}\left(\frac{L_{E}}{10^{4} \mathrm{~km}}\right)\left(\frac{15 \mathrm{MeV}}{E}\right)^{2},
\end{aligned}
$$

which is much smaller than the size of the WP after spread: $\Delta x_{\text {shift }} \ll \sigma_{\text {spread }}$. One may therefore wonder whether the spread of the WP on their way to the Earth can prevent decoherence. This is not the case, as one can most easily see in momentum space. Here the WP width $\sigma_{E}$ is not changed in the course of propagation. Consequently, the above derivation of the coherence length in momentum space always yields the same result, regardless of WP spreading. As we have shown in Sect. 5.2, a detailed analysis in configuration space leads to the same result as in momentum space. Therefore, we can use the same expression for the coherence length as for the WP of width $\sigma_{x}$ without spread. The equivalence of WP separation and energy averaging relies on an integration over the detection time, which is necessary because it is not known precisely at which moment of time a neutrino is produced. This is equivalent to the assumption of a stationary source needed for deriving the theorems of [20,34] about the indistinguishability of long and short WP. The equivalence 
does not hold in the hypothetical case that the times of both production and detection of a single neutrino are measured.

Thus, in the low-density limit, $\xi \ll 1$, we can use Eqs. (4) and (17) to estimate

$L_{\mathrm{coh}} \simeq(800-1800) \mathrm{km}\left(\frac{E}{15 \mathrm{MeV}}\right)^{2}$.

Consequently, $L_{\mathrm{coh}}$ is of the same order of magnitude as the Earth's radius (or the sizes of its core and mantle).

Using expressions for the length of trajectories in different layers we can find regions of complete decoherence, partial decoherence and complete coherence in the $E-\cos \eta$ plane, where $\eta$ is the nadir angle.

For the mantle-crossing trajectories, $|\cos \eta|<0.83$, the length is given by

$L_{M}=D_{E}|\cos \eta|$,

where $D_{E}=12,742 \mathrm{~km}$ is the diameter of the Earth. Then the condition $L_{M}=L_{\mathrm{coh}}$ and Eqs. (88) and (87) give the upper bound on energy of the coherence-loss region:

$E_{\mathrm{dec}}=48.9 \mathrm{MeV}\left(\frac{\sigma_{x}}{2 \cdot 10^{-11} \mathrm{~cm}}\right)^{1 / 2}|\cos \eta|^{1 / 2}$.

For the lower energy bound of the coherence region defined by the condition $L=0.1 L_{\text {coh }}$ we have

$E=150 \mathrm{MeV}|\cos \eta|^{1 / 2}$.

For the core-crossing trajectories the length of each layer of mantle equals

$L_{M}=R_{E}|\cos \eta|-\frac{L_{C}}{2}$,

where $L_{C}$ is the length of trajectory in the core:

$L_{C}=2 \sqrt{R_{C}^{2}-R_{E}^{2} \sin ^{2} \eta}$.

Here $R_{C}=3570 \mathrm{~km}$ is the radius of the core. Now the upper energy bound on the decoherence region in the core:

$E=48.9 \mathrm{MeV}\left(\frac{\sigma_{x}}{2 \cdot 10^{-11} \mathrm{~cm}}\right)^{1 / 2}\left[\left(\frac{R_{C}^{2}}{R_{E}^{2}}\right)-\sin ^{2} \eta\right]^{1 / 4}$.

For the mantle layers

$E=34.7 \mathrm{MeV}\left[\cos \eta-\left(\frac{R_{C}^{2}}{R_{E}^{2}}-\sin ^{2} \eta\right)^{1 / 2}\right]^{1 / 2}$.

These estimates of energy borders of regions of coherence and decoherence are valid with about $30 \%$ accuracy due to uncertainties in our estimate of $\sigma_{x}$.

In Fig. 3 we show the lengths of different trajectories in the mantle and the core as functions of nadir angle according to Eqs. (88), (91), and (92) together with the coherence lengths for different neutrino energies (the horizontal lines).

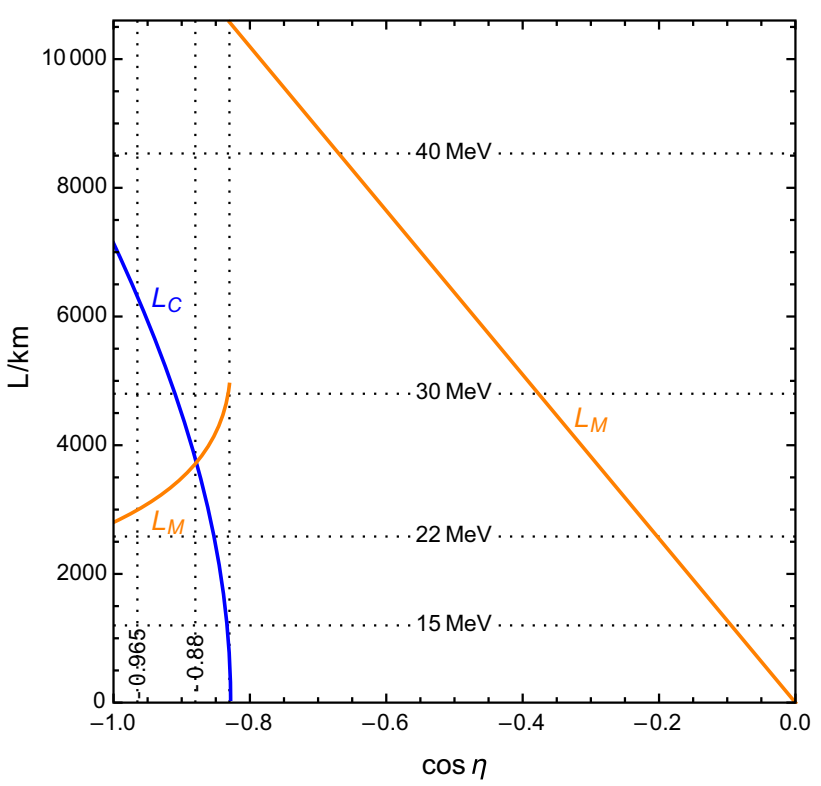

Fig. 3 Lengths of neutrino trajectories in different layers of the Earth as functions of the nadir angle. For $\cos \eta>-0.83, L_{M}$ is the length of the trajectory in the mantle. For $\cos \eta<-0.83$, which corresponds to neutrinos crossing the core and two mantle layers, $L_{M}$ is the length of the trajectory in a single mantle layer. Also shown are the coherence lengths for different neutrino energies (horizontal dotted lines)

Notice that $\sigma_{E} \sim 1 \mathrm{MeV}$ we have found does not depend on neutrino energy, whereas the energy resolution of the detector $\Delta E_{\text {res }} \propto \sqrt{E}$. We find that already at $E>(2-$ 3) $\mathrm{MeV}$ the energy resolution of a detector becomes larger than $\sigma_{E}$, and therefore determines the oscillation pattern apart from the cases of catch-up.

In the following we will describe separately the effects of propagation along the mantle only and along the corecrossing trajectories.

\subsection{Oscillations in the mantle of the Earth}

We consider mixing of two neutrinos with mass splitting $\Delta m_{21}^{2}$. As we discussed before, incoherent fluxes of the mass eigenstates arrive at the Earth and we denote by $c_{0}^{2}$ and $s_{0}^{2}$ the fractions of $v_{1}$ and $v_{2}$. Neutrinos crossing only the Earth's mantle experience one jump in density upon entering the Earth. At this jump the mass states split into eigenstates in the Earth matter and start to oscillate. As we have established in Sect. 4.1, the effect of splitting is determined by change the of the mixing angle due to the density jump. For small densities it is given by Eq. (40), or

$s_{i j} \simeq \frac{1}{2}\left(\xi_{i}-\xi_{j}\right) \sin 2 \theta$.

Here $\sin 2 \theta=\sin 2 \theta_{12} \simeq 0.925$. Since propagation within layers is adiabatic one needs to use the values of $\xi$ immediately before and after a jump. For the $0-1$ jump $\xi_{0} \simeq 0$, and $\xi_{1}$ is given by the density at the surface of the Earth. Numerically, 
$\xi_{1} \simeq 0.0525\left(\frac{E}{15 \mathrm{MeV}}\right)$,

and consequently,

$s_{10} \simeq \frac{1}{2} \xi_{1} \sin 2 \theta=0.0243\left(\frac{E}{15 \mathrm{MeV}}\right)$.

The sine and split effect increase with energy. For $E=$ $30 \mathrm{MeV}$ and $E=60 \mathrm{MeV}$ we have $s_{10}=0.05$ and 0.1 , respectively.

The density jump transforms the mass eigenstates into the eigenstates in the mantle according to

$$
\left(\begin{array}{l}
v_{1} \\
v_{2}
\end{array}\right)=\left(\begin{array}{cc}
c_{10} & s_{10} \\
-s_{10} & c_{10}
\end{array}\right)\left(\begin{array}{l}
v_{1 m}^{(1)} \\
v_{2 m}^{(1)}
\end{array}\right) .
$$

Then the probability to find a $v_{e}$ is

$$
\begin{aligned}
P_{\mathrm{coh}}\left(v_{e}\right)= & c_{0}^{2}\left|c_{1} c_{10}+s_{1} s_{10} e^{i \phi_{1}}\right|^{2} \\
& +s_{0}^{2}\left|s_{1} c_{10} e^{i \phi_{1}}-c_{1} s_{10}\right|^{2},
\end{aligned}
$$

where

$\phi_{1} \simeq \frac{\Delta m_{21}^{2}}{2 E} \int R(\xi(x)) \mathrm{d} x$

is the adiabatic phase acquired along the trajectory.

If the WP of $v_{i m}^{(1)}$ become separated, we obtain

$$
\begin{aligned}
P_{\text {decoh }}\left(v_{e}\right)= & \left(c_{1} c_{0} c_{10}\right)^{2}+\left(c_{1} s_{0} s_{10}\right)^{2}+\left(s_{1} c_{0} s_{10}\right)^{2} \\
& +\left(s_{1} s_{0} c_{10}\right)^{2} .
\end{aligned}
$$

The difference between the complete coherence and loss of coherence cases is

$P_{\mathrm{coh}}\left(v_{e}\right)-P_{\mathrm{decoh}}\left(v_{e}\right)=\frac{1}{2} \cos 2 \theta_{0} \sin 2\left(\theta_{1}-\theta_{0}\right) \sin 2 \theta_{1} \cos \phi_{1}$.

It vanishes when the phase $\phi_{1}$ is averaged, which occurs if a detector has insufficient energy resolution to observe the oscillation pattern.

Inserting Eq. (97) we obtain

$$
\begin{aligned}
& P_{\mathrm{coh}}\left(v_{e}\right)-P_{\mathrm{decoh}}\left(v_{e}\right) \\
& \simeq 0.0243\left(\frac{E}{15 \mathrm{MeV}}\right) \cos 2 \theta_{0} \sin 2 \theta \cos \phi_{1},
\end{aligned}
$$

where we used $\theta_{1} \simeq \theta$. Then the depth of oscillations at $30 \mathrm{MeV}$ can be about $0.05 \cos 2 \theta_{0}$. In the case of normal mass ordering [1]

$\cos 2 \theta_{0}=\frac{F_{\mu}-F_{\tau}}{F_{\mu}}$.

If the fluxes of $v_{\mu}$ and $v_{\tau}$ which arrive at the region of MSW transitions are equal, $\cos 2 \theta_{0}=0$ and no oscillations are expected.
For antineutrinos we have

$\cos 2 \theta_{0}=\frac{\bar{F}_{e}-\bar{F}_{\mu}}{\bar{F}_{e}}$,

and it is expected to be small: of the order 0.1. The fraction can be larger in the high-energy tail where the fluxes decrease exponentially. In the case of inverted mass hierarchy the effect is zero in the antineutrino channel but non-zero in the neutrino channel with $\cos 2 \theta_{0}$ as in Eq. (105) but with larger difference of the neutrino fluxes. So, one can expect few $1-3 \%$ depth of oscillations.

\subsection{Oscillations of neutrinos crossing the core}

Neutrinos that cross the core of the Earth encounter three layers: mantle, core, and mantle. Thus, they pass three jumps of density: at the surface when entering the Earth, when entering the core and when leaving the core. Therefore, each mass state arriving the Earth splits into $2^{3}=8$ components reaching a detector.

The change of mixing in the 0-1 jump is given in Eq. (97). For the 1-2 jump one should use the densities at the surface of the core and in the deepest point of the mantle. This gives

$s_{21} \simeq \frac{1}{2} \sin 2 \theta\left(\xi_{2}-\xi_{1}\right) \simeq 0.0347\left(\frac{E}{15 \mathrm{MeV}}\right)$.

For $E=30 \mathrm{MeV}$ and $E=60 \mathrm{MeV}$ we obtain $s_{21}=0.07$ and 0.14 . In the resonance region the change of the angles is small since already the vacuum 1-2 mixing is large. For larger energies the size of the layers $(\sim 5000-6000 \mathrm{~km})$ becomes smaller than the coherence length (87) and loss of coherence can be neglected (see Fig. 3).

As the two mantle layers are approximately symmetric, for the mixing changes we have $c_{32}=c_{21}, s_{32}=-s_{12}$, $\theta_{1}=\theta_{3}$ and for the phases $\phi_{1}=\phi_{3}$. Then the probability of the $v_{1} \rightarrow v_{e}$ transition in three layers in the coherent case equals

$$
\begin{aligned}
P_{\mathrm{coh}} & \left(\nu_{1} \rightarrow v_{e}\right) \\
= & \mid\left(c_{10} c_{21}^{2}-s_{10} s_{21} c_{21} e^{i \phi_{1}}+c_{10} s_{21}^{2} e^{i \phi_{2}}\right. \\
& \left.+s_{10} s_{21} c_{21} e^{i\left(\phi_{1}+\phi_{2}\right)}\right) c_{3}+\left(-c_{10} s_{21} c_{21} e^{i \phi_{1}}+s_{10} s_{21}^{2} e^{i 2 \phi_{1}}\right. \\
& \left.+c_{10} s_{21} c_{21} e^{i\left(\phi_{1}+\phi_{2}\right)}+s_{10} c_{21}^{2} e^{i\left(2 \phi_{1}+\phi_{2}\right)}\right)\left.s_{3}\right|^{2},
\end{aligned}
$$

where $c_{3}=c_{1}$ is the cosine of the mixing angle in matter in layer 3 .

Let us give an interpretation of the eight terms in Eq. (107), which correspond to eight different channels (chains of transitions) and to $8 \mathrm{WP}$ arriving at the detector (Fig. 4). The terms in the first line describe the whole three layer transition $v_{1} \rightarrow v_{1 m}^{(3)}\left(=v_{1 m}^{(1)}\right)$ with intermediate states in layers 1 (mantle) and 2 (core): 


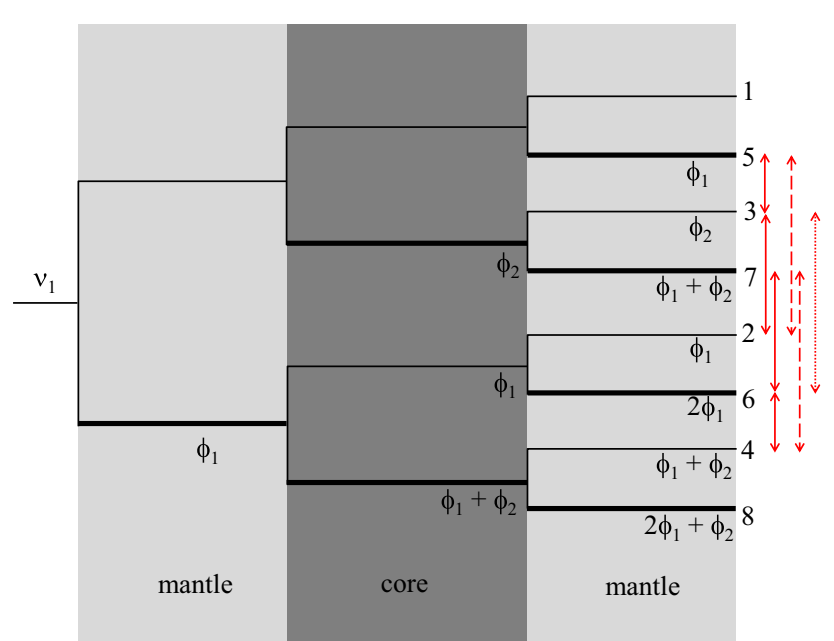

Fig. 4 Scheme of the neutrino state splitting in a medium with three layers, which is realized for core-crossing trajectories in the Earth. Thin (thick) lines correspond to components of states which propagate with high (low) group velocity. Indicated are the phase differences acquired by different components. The numbers on the right-hand side indicate terms in Eq. (107). The catch-up effect can occur between states with equal phases. These states are connected by vertical lines with arrows. Solid, dashed, and dotted lines correspond to the second, first, and third terms in Eq. (109)

$v_{1 m}^{(1)} \rightarrow v_{1 m}^{(2)}, \quad v_{2 m}^{(1)} \rightarrow v_{1 m}^{(2)}, \quad v_{1 m}^{(1)} \rightarrow v_{2 m}^{(2)}, \quad v_{2 m}^{(1)} \rightarrow v_{2 m}^{(2)}$

The state $v_{1 m}^{(3)}=v_{1 m}^{(1)}$ propagates in the third (mantle) layer being then projected onto $v_{e}$. The four terms in the second line of Eq. (107) correspond to the whole transition $v_{1} \rightarrow v_{2 m}^{(3)}=$ $v_{2 m}^{(1)}$ with the same intermediate states and transitions in layers 1 and 2 as in (108). Now the state $v_{2 m}^{(3)}=v_{2 m}^{(1)}$ propagates in the third layer being then projected onto $v_{e}$ in a detector. Any transition $v_{2 m}^{(i)} \leftrightarrow v_{1 m}^{(j)}$ at the density jumps leads to the sine of the difference of the angles, $\left|s_{j i}\right|$, which is small. A minus sign is associated to the transitions $v_{2 m}^{(1)} \rightarrow v_{1 m}^{(2)}$ and $v_{1 m}^{(2)} \rightarrow v_{2 m}^{(1)}$. Every appearance (propagation) of $v_{2 m}^{(i)}$ in the chain of transitions leads to the oscillation phase $\phi_{i}$ since for definiteness we attach the phase factor to the state $v_{2 m}^{(i)}$ in each layer. The state $v_{2 m}^{(i)}$ (with larger mass) moves more slowly than $v_{1 m}^{(i)}$, it arrives later and has the bigger phase.

The first term in Eq. (107) with the amplitude $c_{10} c_{21}^{2}$ and without phase factor corresponds to the fastest component of the state: in all three layers it corresponds to $v_{1 m}^{(i)}$. Notice that this term has the largest amplitude. In contrast, the last term, with the largest phase, $2 \phi_{1}+\phi_{2}$ and the amplitude $s_{10} c_{21}^{2}$ corresponds to the slowest component when the heaviest component $v_{2 m}^{(i)}$ propagates in all three layers. The WPs which correspond to these components cannot produce catchup effect with other components.

The interference terms in the probability (107) are proportional to cosines of all possible differences of the phases.
This includes $0, \phi_{1}, \phi_{2}, \phi_{1}+\phi_{2}, 2 \phi_{1}, 2 \phi_{1}+\phi_{2}$, and $\phi_{1}-\phi_{2}$, $2 \phi_{1}-\phi_{2}$ and the same combinations with opposite signs.

Let us consider the interfence terms which can show up the "catch-up" effect. The critical energy (corresponding to $\xi_{0}$ ) is roughly $500 \mathrm{MeV}$ in the mantle and $200 \mathrm{MeV}$ in the core. So, the whole spectrum of supernova neutrinos is below the critical energy, and consequently, $\xi_{1}, \xi_{2}<\xi_{0}$. Furthermore, the average values of $\xi_{1}$ and $\xi_{2}$ are much smaller than $\xi_{0}$. In this case the derivatives of phases $d \phi_{1} / d E$ and $d \phi_{2} / d E$ are of the same signs as the signs the phases. Therefore, the cancellation of derivatives (which leads to the "catchup" effect) can be realized for the interference terms with differences of phases: $0, \phi_{1}-\phi_{2}, 2 \phi_{1}-\phi_{2}$. Explicitly, we obtain

$$
\begin{aligned}
P_{\mathrm{coh}}^{\mathrm{int}}\left(v_{e}\right)= & \frac{1}{4} \sin 2 \theta_{10} \sin ^{2} 2 \theta_{21} \sin 2 \theta_{1} \\
& -s_{21}^{3} c_{21} \sin 2\left(\theta_{10}+\theta_{1}\right) \cos \left(\phi_{1}-\phi_{2}\right) \\
& +\frac{1}{2} \sin 2 \theta_{10} s_{21}^{4} \sin 2 \theta_{1} \cos \left(2 \phi_{1}-\phi_{2}\right)
\end{aligned}
$$

Note that in general the first term has the phase factor $\cos \left(\phi_{1}-\phi_{3}\right)$, but in the symmetric case with $\phi_{1}=\phi_{3}$, it equals 1 .

Let us consider the three terms in Eq. (109) in detail, giving their interpretation in configuration space.

1. There are four contributions to the term with phase $\left(\phi_{1}-\right.$ $\left.\phi_{2}\right)$. All of them correspond to the catch-up in the case of two layers considered in Sect. 4.2.

(i) Interference of the third and fifth terms of Eq. (107) corresponds to different motion of the WP in the second (core) and third (mantle) layers: the component $v_{1 m}^{(1)}$ splits into $v_{1 m}^{(2)}$ and $v_{2 m}^{(2)}$ crossing the density jump between the mantle and the core. In the core $v_{1 m}^{(2)}$ propagates faster than $v_{2 m}^{(2)}$. Both components split further when entering the mantle again. Then $v_{1 m}^{(3)}$, which originates from $v_{1 m}^{(2)}$, moves faster than $v_{2 m}^{(3)}$, and therefore its WP can catch up with the packet of $v_{2 m}^{(3)}$ originated from $v_{2 m}^{(2)}$. Catch-up occurs in the third layer.

(ii) Interference of sixth and seventh terms in Eq. (107) is similar to (i) with the only difference that the two interfering channels originate from $v_{2 m}^{(1)}$ (in the first layer).

(iii) In the case of interference of third and second terms in Eq. (107) (in contast to (ii)) the first (mantle) and the second (core) layers are involved. The catch-up occurs in the core; in the third layer $v_{1 m}^{(3)}$ propagates in both channels, so neither shift nor phase difference are acquired. The consideration is similar to the previous case. 
(iv) Interference of fourth and sixth terms is similar to case (iii) with the only difference that in the third layer in both channels $v_{2 m}^{(3)}$ propagates.

According to Eq. (109) this term is suppressed by $s_{21}^{3}$. Let us consider the condition of coherence restoration, which in the case of small density is given by Eq. (53). In the first approximation the condition is reduced to equality, $L_{M} \simeq L_{C}$. According to Eqs. (91) and (92) this condition is satisfied for the nadir angle $\eta$ given by

$$
\cos \eta \simeq \frac{3}{2 \sqrt{2}} \sqrt{1-\frac{R_{C}^{2}}{R_{E}^{2}}}=\frac{3}{\sqrt{2}} \cos \eta_{c} \simeq 0.89
$$

where $R_{C}$ and $R_{E}$ are the radii of the core and the Earth; $\eta_{C}$ is the nadir angle of the trajectory which touches the core, $\cos \eta_{c}=0.836$. The value $\cos \eta=0.89$ gives $L_{m}=$ $3760 \mathrm{~km}$.

2. The term with phase $\left(2 \phi_{1}-\phi_{2}\right)$ in Eq. (109) originates from the interference of the third and sixth terms of Eq. (107). In turn these terms are due to the chains of transitions

$$
v_{1 m}^{(1)} \rightarrow v_{2 m}^{(2)} \rightarrow v_{1 m}^{(1)}, \quad v_{2 m}^{(1)} \rightarrow v_{1 m}^{(2)} \rightarrow v_{2 m}^{(1)}
$$

This interference is a genuine 3-layer effect: In the first channel of (111) the WP is faster in the first mantle layer, then slower in the core, then again faster in the second mantle layer. In the second channel of (111) inversely: the WP moves first more slowly, then faster, then again more slowly. This corresponds to the change of subscript indices of neutrino states in (111). So, the order of WP in configuration space changes twice (the WP of the first channel arrives first at the core, in the core the second WP overtakes the first one) and catch-up occurs in the third layer.

The coherence restoration condition for this interference term is $2 L_{M} \simeq L_{C}$, which can be realized according to (91) and (92) for

$$
\cos \eta \simeq \frac{2}{\sqrt{3}} \sqrt{1-\frac{R_{c}^{2}}{R_{E}^{2}}}=\frac{2}{\sqrt{3}} \cos \eta_{c} \simeq 0.965
$$

In this case $L_{C}=3100 \mathrm{~km}$. Unfortunately, the amplitude of this interference term is suppressed very strongly by $s_{21}^{4}$. Equations (110) and (112) are conditions for complete overlap of WP; partial overlap of WP and partial catchup can be realized in a wider region of nadir angles and neutrino energies.

3. The first term in Eq. (109) does not contain phases, but we keep it since it also shows the catch-up effect. The effect, however, does not depend on energy and therefore it does not change by averaging over the energy and is present also in the incoherent case. There are two contribution to this term: interference of the second and fifth terms in Eq. (107), which both have the same phase $\phi_{1}$, and interference of the fourth and seventh terms with common phase $\phi_{1}+\phi_{2}$. In the case of 2-5 interference one WP moves more slowly in the third layer, whereas the other WP moves more slowly in the first layer (they move with the same high speed in the core). So the second WP catches up with the first one in the third layer. The same is the case for 4-7 interference with the only difference that in the core both WP move with the same small speed. Here the catch-up does not depend on energy (if we neglect the energy dependence of the depth of interference). The derivatives of the phases are the same for both channels, and therefore there is no averaging over the energy and the catch-up is complete.

This term is proportional to $s_{21}^{2}$ and thus less suppressed than the others. It would acquire a phase if the third layer was different from the first one. However, in this case the coherence condition would be satisfied when $L_{3} \simeq L_{1}$.

The amplitudes of the catch-up effect can be compared with the main term in Eq. (107), $\left(c_{10} c_{21} c_{1}\right)^{2}$, and with the depth of the main oscillatory term (without loss of coherence). The latter appears due to interference of the first term in Eq. (107) with the fifth, seventh, and eighth terms, which contain $s_{21}$ and $s_{10}$ to the first power. The depths equal $0.5 \sin 2 \theta_{10} \sin 2 \theta_{3}, 0.5 \sin 2 \theta_{21} \sin 2 \theta_{3} \simeq 0.08$ for $E=15 \mathrm{MeV}$.

The Earth matter effect and in particular the catch-up effect are further suppressed because of the presence of the $v_{2}$ component in the arriving supernova neutrino flux. For the $v_{2} \rightarrow v_{e}$ transition the probability can be obtained from the previous result by the substitutions $c_{10} \rightarrow-s_{10}, s_{10} \rightarrow c_{10}$. As a result, all the interference terms Eq. (109) change sign, so the observable effect will be proportional to the difference of the $v_{1}$ and $v_{2}$ fluxes, $F\left(v_{1}\right)-F\left(v_{2}\right)$. In turn, this difference is determined by the dynamics of flavor transitions in the supernova. An additional suppression can be estimated by a factor $0.1-0.3$.

Taking $E=15 \mathrm{MeV}, \rho=5 \mathrm{~g} \mathrm{~cm}^{-3}$, and $\rho=11 \mathrm{~g} \mathrm{~cm}^{-3}$ for the densities of the Earth's mantle and core, respectively, we have $\theta_{1} \simeq 36^{\circ}$ and $\theta_{2} \simeq 39^{\circ}$. Consequently, $\theta_{10} \simeq 2.0^{\circ}$ and $\theta_{21} \simeq 2.6^{\circ}$. Approximating the energy dependence of combination of mixing parameters by power laws, Eq. (109) becomes roughly

$$
\begin{aligned}
P_{\mathrm{coh}}^{\text {int }}\left(v_{e}\right) \simeq & 1.4 \cdot 10^{-4}\left(\frac{E}{15 \mathrm{MeV}}\right)^{3} \\
& -9 \cdot 10^{-5}\left(\frac{E}{15 \mathrm{MeV}}\right)^{3} \cos \left(\phi_{1}-\phi_{2}\right) \\
& +1.5 \cdot 10^{-7}\left(\frac{E}{15 \mathrm{MeV}}\right)^{5} \cos \left(2 \phi_{1}-\phi_{2}\right)
\end{aligned}
$$


The approximation is only accurate up to about $30 \%$ for $5 \mathrm{MeV}<E<80 \mathrm{MeV}$, but it serves to show that the oscillation depth inside the Earth is small. Consequently, the catchup effect is too small (at the level of $0.1 \%$ at most) to be observable with existing detectors, but it may become relevant in the future, especially if a supernova explodes close to us, producing a huge number of events.

One last comment: as we have established, the separation and spread of the WP have sizes of tens of meters. Such a state passes through the detector during about $t_{\text {state }} \sim 10^{-7} \mathrm{~s}$. So, in principle, present technology allows one to study parts of the packet. The problem is that the time of emission is not known and even the shortest features of the burst are about a few msec, which is much bigger than $t_{\text {state }}$.

\section{Conclusions}

Supernova neutrinos have several unique features: their wave packets (WP) are very short and coherence is lost very quickly during the propagation. They propagate for a long time, so the spread of individual WP can reach macroscopic sizes, up to hundreds of meters for a galactic supernova. Neutrino mass eigenstates arriving at the surface of the Earth split and oscillate again inside the Earth.

1. We have recalculated the size of the WP of supernova neutrinos, finding a uniquely short length of about $10^{-11} \mathrm{~cm}$, which corresponds to an energy spread $\sigma_{E} \sim$ $1 \mathrm{MeV}$ - not much smaller than the neutrino energy itself. $\sigma_{E}$ does not depend on neutrino energy and is approximately the same for all neutrino species in all phases of the supernova.

2. The coherence length is smaller than $100 \mathrm{~km}$ for the $1-$ 3 mass splitting and of order $1000 \mathrm{~km}$ for the 1-2 mass splitting. The separation of the WP arriving at the Earth from a supernova in the galactic center can be as large as $40 \mathrm{~m}$ for the 1-2 mass splitting and $E=15 \mathrm{MeV}$.

3. Each wave packet spreads due to the presence of different energy components in it. The spread is proportional to the neutrino mass squared and can reach a macroscopic size. Usually the separation of the packets is bigger that the spread.

An exceptional situation is realized for neutrinos oscillating in the Earth. Here oscillations occur due to the interference of components originating from the split of the WP of a mass eigenstate at the surface of the Earth. In this case the spread of the WP is much larger than their relative shift inside the Earth, so they continue to overlap. We have showed, however, that this does not change the coherence condition, which is determined by the original size of the WP without spread.
4. We have explored the coherence condition for supernova neutrinos oscillating in the Earth. The coherence length turns out to be comparable with the sizes of the mantle and the core. Thus, for low energies $(E<30 \mathrm{MeV})$ coherence is completely lost for most nadir angles. For a large range of energies and nadir angles the loss of coherence is partial. Only for high energies and shallow trajectories decoherence can be neglected.

5. We have studied oscillations in a multi-layer medium characterized by the adiabatic change of density within layers and sudden jumps of density between layers. This has applications for neutrinos crossing the shock waves in a supernova and for neutrinos propagating inside the Earth along core-crossing trajectories.

6. A splitting of eigenstates occurs at each density jump, so for two-neutrino mixing $2^{n}$ components are formed after $n$ crossings. This multiplication has no meaning if the shift of the WP within each layer can be neglected. In this case the problem is reduced to the two-neutrino problem. However, if the shift and therefore decoherence are substantial, the multiple splitting has physical sense and can lead to new phenomena.

In particular, it leads to the new interesting phenomenon of the partial restoration of coherence due to a "catch-up effect". In other words, in a multi-layer medium coherence can be partially restored and the coherence length can be increased beyond the usual estimate. In the simplest realization, for two layers, this happens if a component of the WP that travels faster through layer 1 arrives at a detector at the same time as a component that was slower in layer 1 but faster in layer 2 . The described effect yields corrections at the percent level or below for supernova neutrinos oscillating in Earth matter, but, in principle, it can be observed if a very high-statistics signal from a close supernova is detected.

7. We have studied decoherence in parallel in configuration and momentum space, checking the equivalence between both representations. Although at first sight the catch-up effect seems to depend on the size of the WP, this is not the case in the examples we have considered. We have explicitly shown how this size-independence is due to the restoration of overlap of WP, confirming the general (abstract) results of [20] and [34]. In a sense the catch-up effect is a non-trivial effect of the averaging of oscillation probabilities over energy.

We have verified that in all cases we have studied there is an equivalence between configuration and momentum space. That is, we can choose to do all calculations either with WP that separate or by suitably averaging over energy. The observable oscillatory picture is determined by the initial energy spectrum, the energy resolution of the detector, and by the phase acquired between source 
and detector as a function of energy, unless time tagging is arranged.

Acknowledgments We would like to thank Evgeny Akhmedov, Yasaman Farzan, Carlo Giunti, Alessandro Mirizzi, Georg Raffelt, Günter Sigl, Irene Tamborra, Ricard Tomas Bayo, and Mariam Tortola Baixauli for helpful discussions. We acknowledge support from the European Union FP7 ITN Invisibles (Marie Curie Actions, PITN-GA-2011289442) and from the Max Planck fellowship M.FW.A.KERN0001.

Open Access This article is distributed under the terms of the Creative Commons Attribution 4.0 International License (http://creativecomm ons.org/licenses/by/4.0/), which permits unrestricted use, distribution, and reproduction in any medium, provided you give appropriate credit to the original author(s) and the source, provide a link to the Creative Commons license, and indicate if changes were made.

Funded by SCOAP ${ }^{3}$.

\section{References}

1. A.S. Dighe, A.Y. Smirnov, Identifying the neutrino mass spectrum from a supernova neutrino burst. Phys. Rev. D 62, 033007 (2000). arXiv:hep-ph/9907423

2. J.T. Pantaleone, Neutrino oscillations at high densities. Phys. Lett. B 287, 128-132 (1992)

3. S. Samuel, Neutrino oscillations in dense neutrino gases. Phys. Rev. D 48, 1462-1477 (1993)

4. J.T. Pantaleone, Neutrino flavor evolution near a supernova's core. Phys. Lett. B 342, 250-256 (1995). arXiv:astro-ph/9405008

5. S. Pastor, G.G. Raffelt, D.V. Semikoz, Physics of synchronized neutrino oscillations caused by self-interactions. Phys. Rev. D 65 , 053011 (2002). arXiv:hep-ph/0109035

6. H. Duan, G.M. Fuller, Y.-Z. Qian, Collective neutrino flavor transformation in supernovae. Phys. Rev. D 74, 123004 (2006). arXiv:astro-ph/0511275

7. S. Hannestad, G.G. Raffelt, G. Sigl, Y.Y.Y. Wong, Self-induced conversion in dense neutrino gases: Pendulum in flavour space. Phys. Rev. D 74, 105010 (2006). arXiv:astro-ph/0608695. [Erratum-ibid. D 76, 029901 (2007)]

8. H. Duan, G.M. Fuller, J. Carlson, Y.-Z. Qian, Simulation of coherent nonlinear neutrino flavor transformation in the supernova environment: correlated neutrino trajectories. Phys. Rev. D 74, 105014 (2006). arXiv:astro-ph/0606616

9. G.G. Raffelt, A.Y. Smirnov, Self-induced spectral splits in supernova neutrino fluxes. Phys. Rev. D 76, 081301 (2007). arXiv:0705.1830 [hep-ph]. [Erratum-ibid. D 77, 029903 (2008)]

10. G.L. Fogli, E. Lisi, A. Marrone, A. Mirizzi, Collective neutrino flavor transitions in supernovae and the role of trajectory averaging. JCAP 0712, 010 (2007). arXiv:0707.1998 [hep-ph]

11. G.G. Raffelt, A.Y. Smirnov, Adiabaticity and spectral splits in collective neutrino transformations. Phys. Rev. D 76, 125008 (2007). arXiv:0709.4641 [hep-ph]

12. G.G. Raffelt, Self-induced parametric resonance in collective neutrino oscillations. Phys. Rev. D 78, 125015 (2008). arXiv:0810.1407 [hep-ph]

13. H. Duan, J.P. Kneller, Neutrino flavour transformation in supernovae. J. Phys. G 36, 113201 (2009). arXiv:0904.0974 [astroph.HE]

14. H. Duan, G.M. Fuller, Y.-Z. Qian, Collective neutrino oscillations. Ann. Rev. Nucl. Part. Sci. 60, 569-594 (2010). arXiv:1001.2799 [hep-ph]

15. A. Mirizzi et al., Supernova neutrinos: production, oscillations and detection. Rivista del Nuovo Cimento 39(1-2), 1-112 (2016). arXiv:1508.00785 [astro-ph.HE]
16. K.M. Patton, J.P. Kneller, G.C. McLaughlin, Stimulated neutrino transformation through turbulence on a changing density profile and application to supernovae. Phys. Rev. D 91, 025001 (2015). arXiv:1407.7835 [hep-ph]

17. H. Anada, H. Nishimura, Neutrino vacuum oscillation and neutrino burst from SN1987a. Phys. Rev. D 37, 552-554 (1988)

18. H. Anada, H. Nishimura, Coherence condition for resonant neutrino oscillation. Phys. Rev. D 41, 2379-2383 (1990)

19. B. Kayser, On the quantum mechanics of neutrino oscillation. Phys. Rev. D 24, 110-116 (1981)

20. K. Kiers, S. Nussinov, N. Weiss, Coherence effects in neutrino oscillations. Phys. Rev. D 53, 537-547 (1996). arXiv:hep-ph/9506271

21. W. Grimus, P. Stockinger, S. Mohanty, Field-theoretical approach to coherence in neutrino oscillations. Phys. Rev. D 59, 013011 (1999). arXiv:hep-ph/9807442

22. C.Y. Cardall, Coherence of neutrino flavor mixing in quantum field theory. Phys. Rev. D 61, 073006 (2000). arXiv:hep-ph/9909332

23. C. Giunti, C.W. Kim, Quantum mechanics of neutrino oscillations. Found. Phys. Lett. 14, 213-229 (2001). arXiv:hep-ph/0011074

24. T. Ohlsson, Equivalence between neutrino oscillations and neutrino decoherence. Phys. Lett. B 502, 159-166 (2001). arXiv:hep-ph/0012272 [hep-ph]

25. C. Giunti, Coherence and wave packets in neutrino oscillations. Found. Phys. Lett. 17, 103-124 (2004). arXiv:hep-ph/0302026

26. E.Kh. Akhmedov, A.Y. Smirnov, Paradoxes of neutrino oscillations. Phys. Atom. Nucl. 72, 1363-1381 (2009). arXiv:0905.1903 [hep-ph]

27. E.Kh. Akhmedov, D. Hernandez, A.Yu. Smirnov, Neutrino production coherence and oscillation experiments. JHEP 04, 052 (2012). arXiv: 1201.4128 [hep-ph]

28. S.P. Mikheyev, A.Yu. Smirnov, Resonant neutrino oscillations in matter. Prog. Part. Nucl. Phys. 23, 41-136 (1989)

29. J.T. Peltoniemi, V. Sipilainen, Neutrino propagation in matter using the wave packet approach. JHEP 06, 011 (2000). arXiv:hep-ph/0004162

30. S. Hollenberg, H. Päs, Adiabatic and nonadiabatic perturbation theory for coherence vector description of neutrino oscillations. Phys. Rev. D 85, 013013 (2012). arXiv:1103.5949 [hep-ph]

31. R.L.N. Oliveira, M.M. Guzzo, P.C. de Holanda, Solar neutrinos do not bound the decoherence effect. arXiv:1408.0823 [hep-ph]

32. G.G. Raffelt, I. Tamborra, Synchronization versus decoherence of neutrino oscillations at intermediate densities. Phys. Rev. D 82, 125004 (2010). arXiv: 1006.0002 [hep-ph]

33. C. Giunti, C.W. Kim, U.W. Lee, When do neutrinos cease to oscillate? Phys. Lett. B 421, 237-244 (1998). arXiv:hep-ph/9709494

34. L. Stodolsky, When the wavepacket is unnecessary. Phys. Rev. D 58, 036006 (1998). arXiv:hep-ph/9802387

35. J. Kersten, Coherence of supernova neutrinos. in Proceedings of the Neutrino Oscillation Workshop 2012, ed. by P. Bernardini, G. Fogli, E. Lisi. Vol. 237-238 of Nucl. Phys. Proc. Suppl. (Elsevier, Amsterdam, 2013), pp. 342-344

36. E. Akhmedov, J. Kopp, M. Lindner, Decoherence by wave packet separation and collective neutrino oscillations. arXiv:1405.7275 [hep-ph]

37. M. Beuthe, Oscillations of neutrinos and mesons in quantum field theory. Phys. Rept. 375, 105-218 (2003). arXiv:hep-ph/0109119

38. Y.-L. Chan, M.-C. Chu, K. M. Tsui, C. F. Wong, J. Xu, Wavepacket treatment of neutrino oscillations and its implications on determining the neutrino mass hierarchy. arXiv:1507.06421 [hep$\mathrm{ph}]$

39. C. Giunti, Neutrino wave packets in quantum field theory. JHEP 11, 017 (2002). arXiv:hep-ph/0205014

40. I. Tamborra, G.G. Raffelt, L. Hüdepohl, H.-T. Janka, Impact of eV-mass sterile neutrinos on neutrino-driven supernova outflows. JCAP 1201, 013 (2012). arXiv:1110.2104 [astro-ph.SR] 
41. T. Fischer, G. Martínez-Pinedo, M. Hempel, M. Liebendörfer, Neutrino spectra evolution during proto-neutron star deleptonization. Phys. Rev. D 85, 083003 (2012). arXiv:1112.3842 [astro-ph.HE]

42. M.T. Keil, G.G. Raffelt, H.-T. Janka, Monte Carlo study of supernova neutrino spectra formation. Astrophys. J. 590, 971-991 (2003). arXiv:astro-ph/0208035

43. C.Y. Cardall, Theory of neutrino flavor mixing. arXiv:hep-ph/0107004

44. S. Nussinov, Solar neutrinos and neutrino mixing. Phys. Lett. B 63, 201-203 (1976)
45. P.C. de Holanda, A.Yu. Smirnov, Solar neutrinos: the SNO salt phase results and physics of conversion. Astropart. Phys. 21, 287301 (2004). arXiv:hep-ph/0309299

46. M.C. Gonzalez-Garcia, M. Maltoni, T. Schwetz, Updated fit to three neutrino mixing: status of leptonic CP violation. JHEP 1411, 052 (2014). arXiv:1409.5439 [hep-ph]

47. J. Xu, L.-J. Hu, R.-C. Li, X.-H. Guo, B.-L. Young, Effects of shock waves on neutrino oscillations in three supernova models. arXiv:1412.7240 [hep-ph] 